\title{
Credit Rating Score Analysis
}

\author{
Wolfgang Karl Härdle \\ Phoon Kok Fai \\ David Lee Kuo Chuen
}

\section{$\underline{v}$

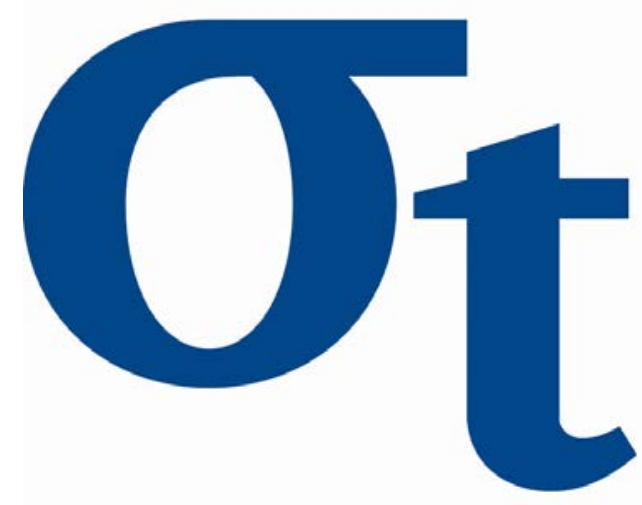

* Humboldt-Universität zu Berlin, Germany *2 Singapore Management University, Singapore

This research was supported by the Deutsche Forschungsgemeinschaft through the SFB 649 "Economic Risk".

\author{
http://sfb649. wiwi.hu-berlin.de \\ ISSN 1860-5664
}




\title{
Credit Rating Score Analysis *
}

\author{
Wolfgang Karl Härdle ${ }^{1,2}$, Phoon Kok Fai ${ }^{2}$, and David Lee Kuo Chuen ${ }^{2}$ \\ ${ }^{1}$ Ladislaus von Bortkiewicz Chair of Statistics, C.A.S.E. - Center for Applied Statistics and \\ Economics, Humboldt-Universitt zu Berlin, Unter den Linden 6, 10099 Berlin, Germany \\ ${ }^{2}$ School of Business, Singapore Management University, 50 Stamford Road, Singapore 178899
}

November 2, 2016

\begin{abstract}
We analyse a sample of funds and other securities each assigned a total rating score by an unknown expert entity. The scores are based on a number of risk and complexity factors, each assigned a category (factor score) of Low, Medium, or High by the expert entity. A principal component analysis of the data reveals that based on the chosen risk factors alone we cannot identify a single underlying latent source of risk in the data. Conversely, the chosen complexity factors are clearly related to one or two underlying sources of complexity. For the sample we find a clear positive relation between the first principal component and the total expert score. An attempt to match the securities' expert score by linear projection of their individual factor scores yields a best case correlation between expert score and projection of 0.9952 . However, the sum of squared differences is, at 46.5552 , still notable.
\end{abstract}

JEL classification: C01, G00, G17, G24

Keywords: Credit risk, Principal Components Analysis, Credit Rating Score

*Financial support from Deutsche Forschungsgemeinschaft (DFG) via CRC 649 "Economic Risk" is gratefully acknowledged. 


\section{Introduction}

We are provided with a sample of $n=100$ funds and other securities that have been assigned a rating score by an unknown expert entity - the expert (rating) score in the following. We assume the rating score to depend on a set of six risk factors and five complexity factors, each modelled as random variables on an ordinal scale of Low, Medium, High. The risk factors are volatility, liquidity, credit rating, duration / cash flow, leverage, and diversification degree. The complexity factors comprise of the number of structural layers, expansiveness of derivatives, availability \& known pricing models, number of return outcome scenarios, and transparency / ease of understanding. In addition to the rating score, we know the category (i.e. Low, Medium, High) assigned to each factor for any given security included in the sample. Figure 1 and Figure 2 show histograms for each of the risk and complexity factors, respectively.
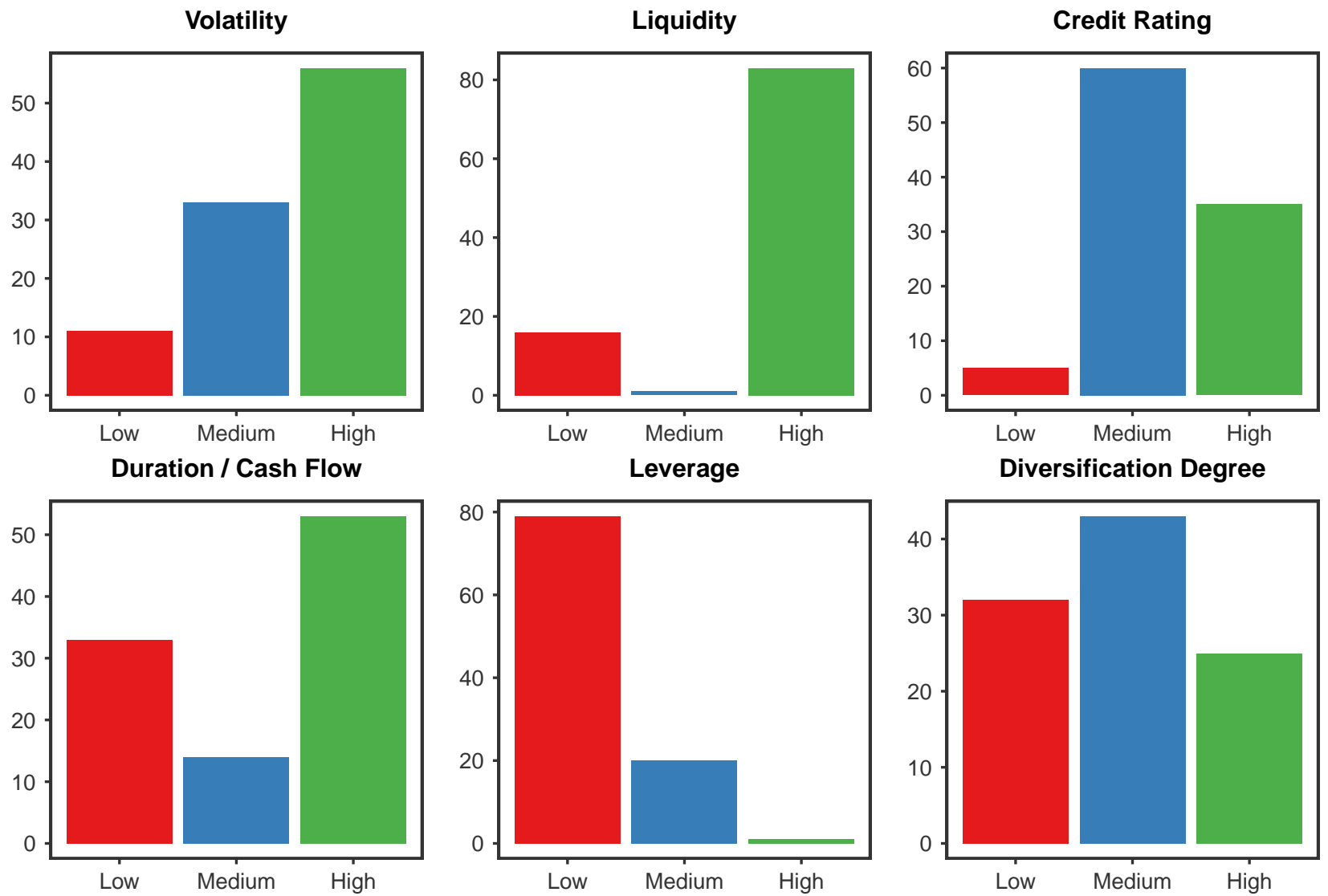

Figure 1: Histograms of risk factor scores

To get a better impression regarding the relation between individual securities in the sample, we perform cluster analyses based on i) only the risk factors, ii) only the complexity factors, and iii) both risk and complexity factors in the sample. In particular, we apply the Ward clustering algorithm using an Euclidean distance matrix. This algorithm is chosen to ensure that individual clusters are as homogenous as possible. However, other algorithms such as the single linkage or complete linkage algorithms can be applied as well (Härdle and Simar, 2015, Chapter 12). The results are depicted in Figure 3. 
No. of Structural Layers

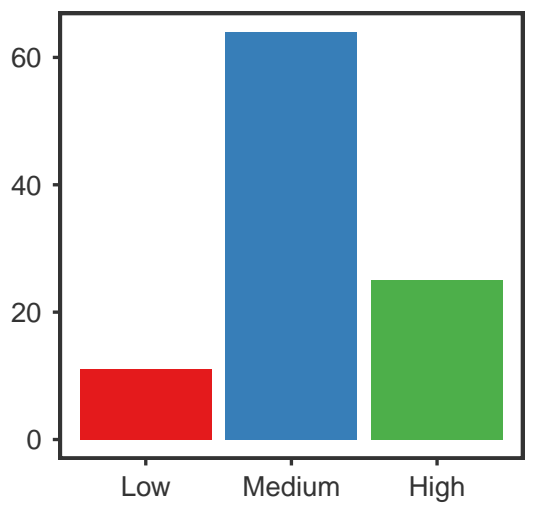

No. of Return Outcome Scenarios

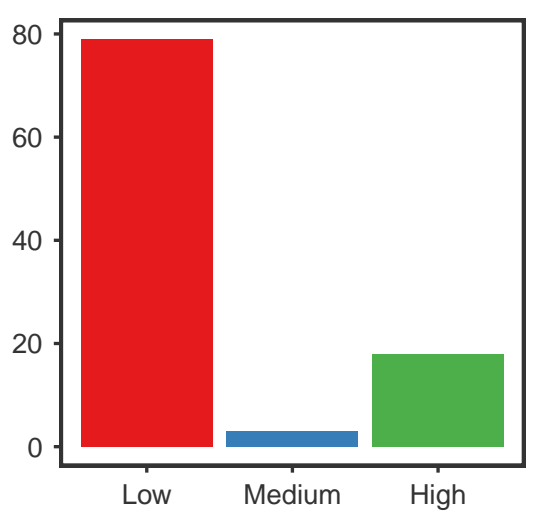

Expansiveness of Derivatives

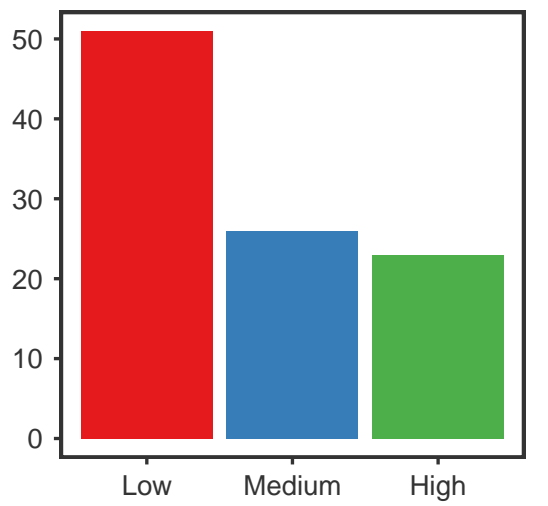

Transparency/ Ease of Understanding

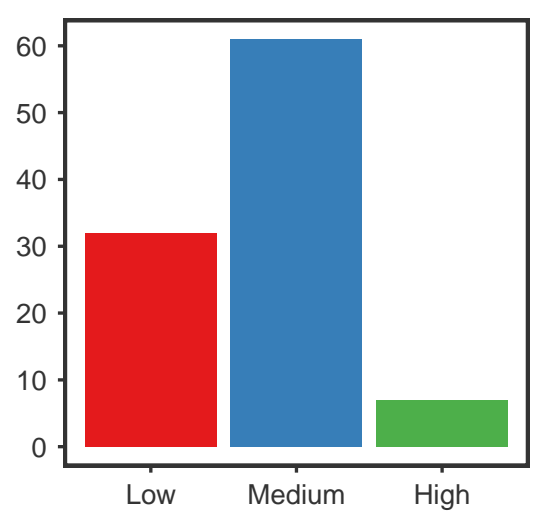

Avail \& Known Valuation Models

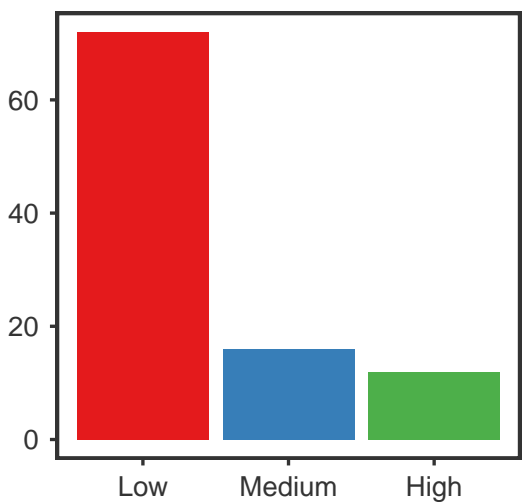

Figure 2: Histograms of complexity factor scores 
Risk

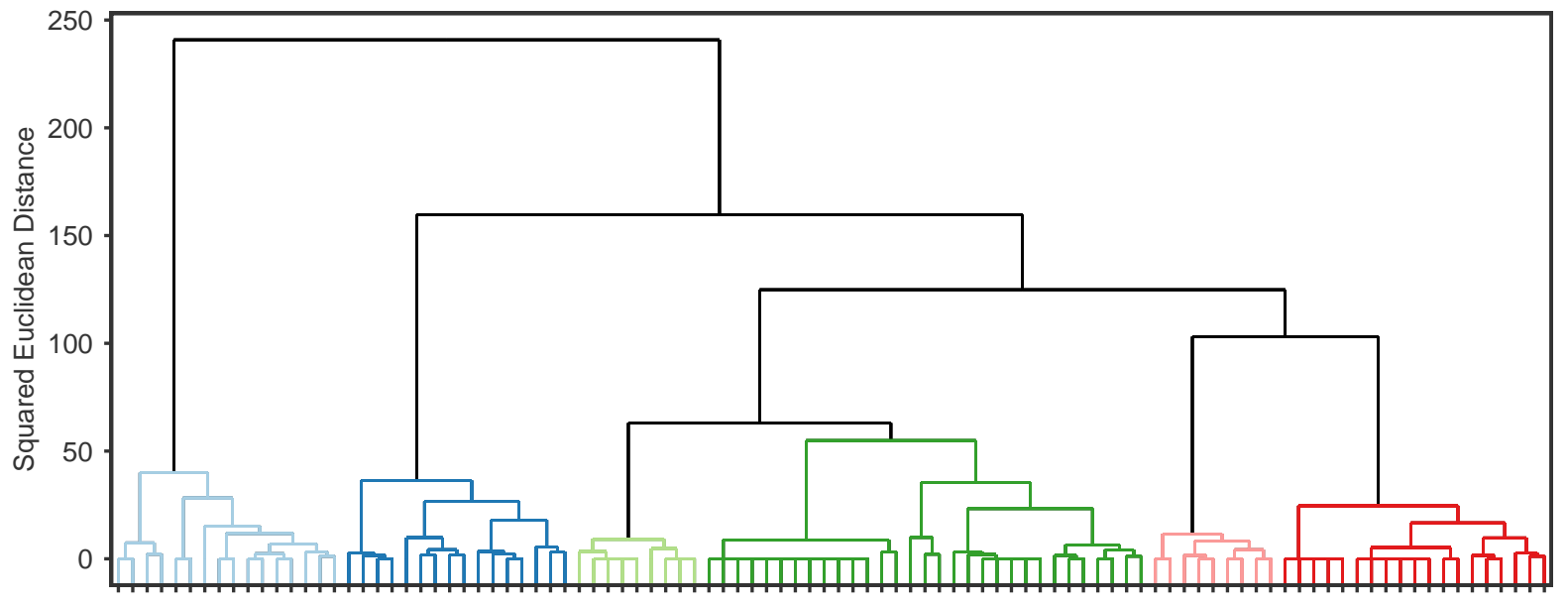

Securities

Complexity

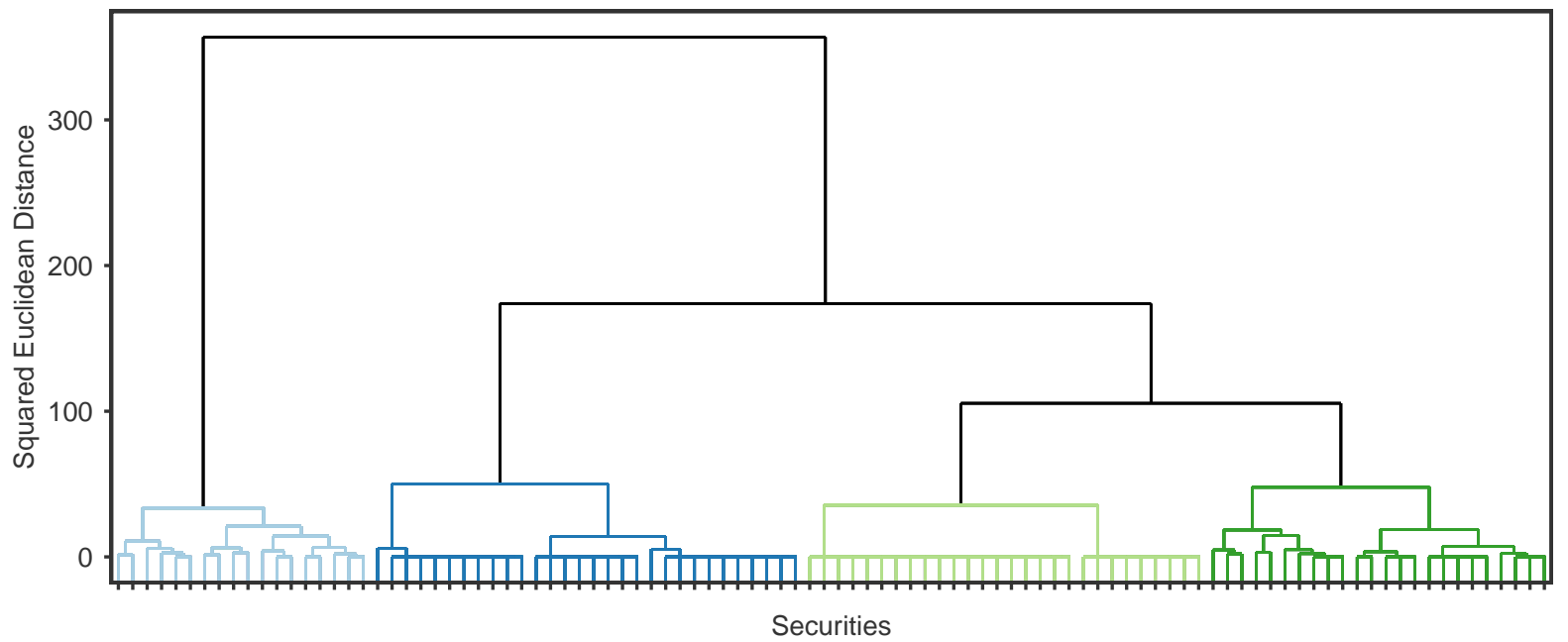

AlI

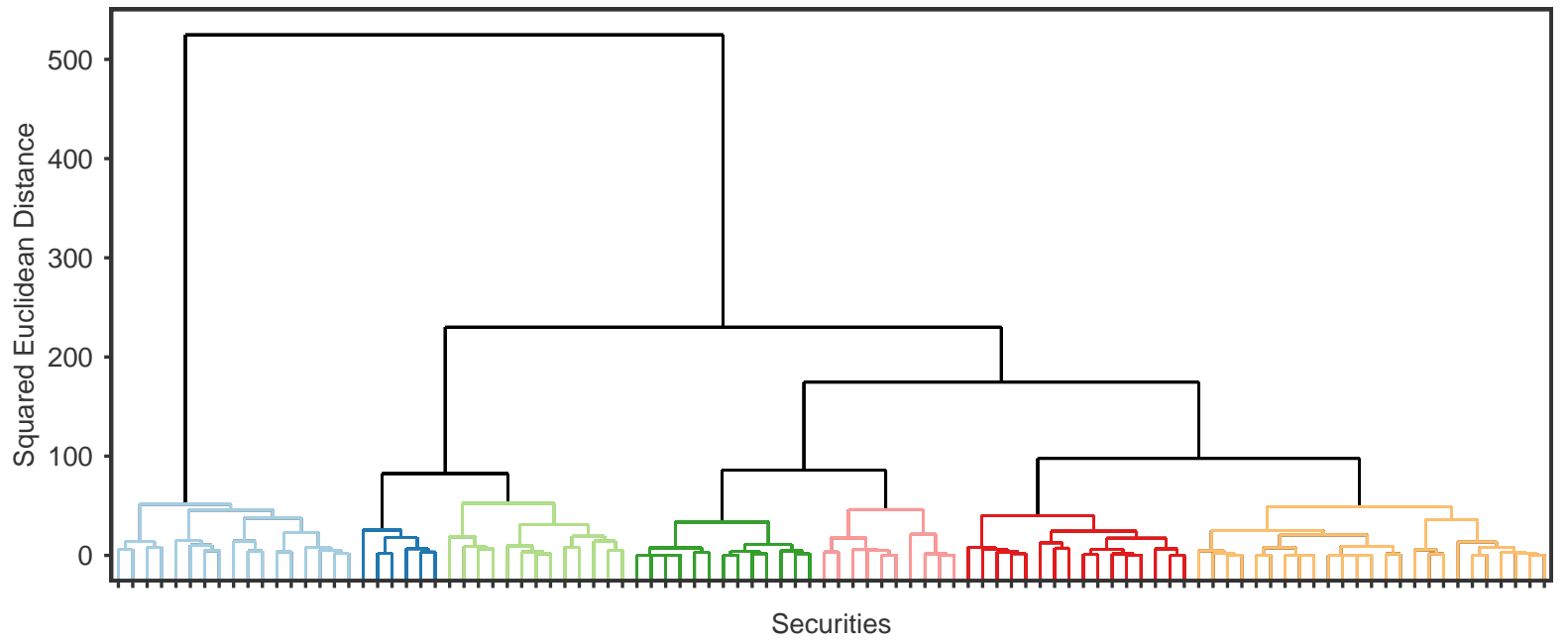

Figure 3: Dendrograms of cluster analysis. Ward algorithm using Euclidean distances. Clusters formed below a threshold of 60 are coloured. 
Risk

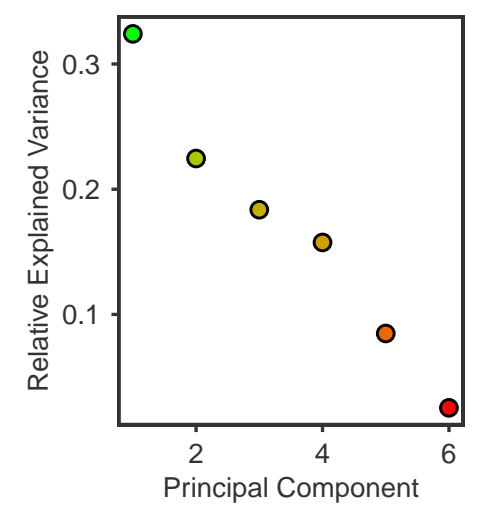

Complexity

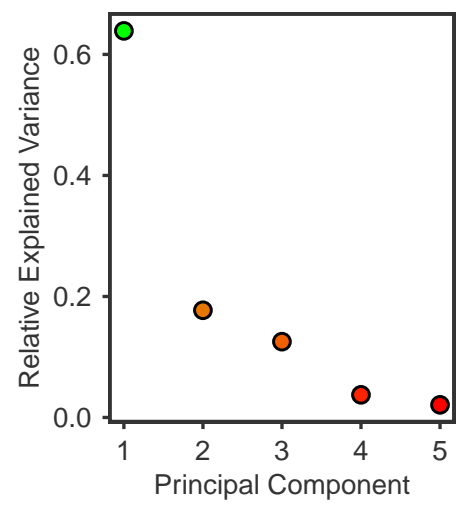

All

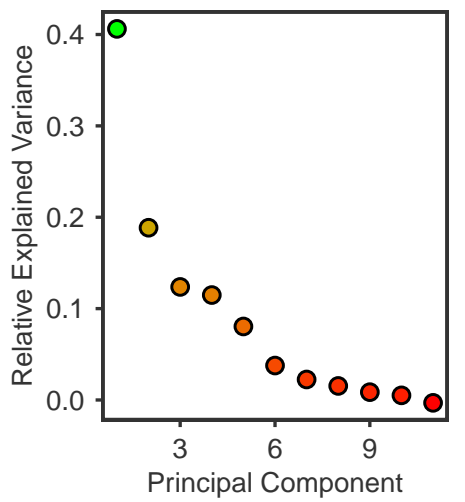

Figure 4: Fraction of variance explained by each of the principal components

\begin{tabular}{c|rrr}
\hline \hline & $X^{\text {Risk }}$ & $X^{\text {Comp }}$ & $X^{\text {All }}$ \\
\hline$w_{1}$ & -0.2141 & 0.3279 & -0.1594 \\
$w_{2}$ & 0.6013 & 0.4030 & 0.4275 \\
$w_{3}$ & 0.0905 & 0.5185 & 0.1237 \\
$w_{4}$ & 0.5106 & 0.4896 & 0.2687 \\
$w_{5}$ & 0.1308 & 0.4707 & -0.1087 \\
$w_{6}$ & 0.5537 & & 0.1166 \\
$w_{7}$ & & & -0.1929 \\
$w_{8}$ & & & -0.3142 \\
$w_{9}$ & & & -0.4440 \\
$w_{10}$ & & & -0.4553 \\
$w_{11}$ & & & -0.3722 \\
\hline \hline
\end{tabular}

Table 1: Projection vectors for $P C_{1}$

Projection vectors for $P C_{1}$ obtained from the eigendecompositions of the polychoric correlation matrices of $X^{R i s k}$, $X^{C o m p}$, and $X^{A l l}$.

\section{Principal Components Analysis of Factor Scores}

Principal components analysis (PCA) allows for the identification of uncorrelated latent factors that drive the variation in a sample of multivariate random variables. We consider a random variable $Y=\left(Y_{1}, \ldots, Y_{j}, \ldots, Y_{k}\right)^{\top}$ with $Y_{j} \in\{$ Low, Medium, High $\}, 1 \leq j \leq k . Y$ represents a vector of the risk and complexity categories assigned to a security $i$ by the expert entity. To later be able to perform PCA on our sample we assign a discrete scale $\{1,2,3\}$ to each $Y_{j}$ yielding a random variable $X=\left(X_{1}, \ldots, X_{j}, \ldots, X_{k}\right)^{\top}$ with $X_{j} \in\{1,2,3\}, 1 \leq j \leq k$ (i.e. $Y_{j}=$ High is equivalent to $X_{j}=3$ ). For easier reference let us refer to each of the $X_{j}$ as a factor score.

Our sample is now represented by a discrete matrix $X \in\{1,2,3\}^{n \times k}$, with each row $i$ representing a security and each column $j$ representing a factor. The element $x_{i, j}$ is therefore security $i$ 's score for the $j$-th factor. We still cannot apply PCA to $X$ directly, however, without violating the basic assumption of normally distributed continuous random variables made in PCA. To circumvent this issue, we apply a discrete PCA using the polychoric correlation matrix of the factor scores (Kolenikov and Angeles, 2009).

Just as the cluster analysis, PCA is performed on three sub-samples of $X ; X^{R i s k}, X^{C o m p}$, and $X^{A l l}$. The number of columns of $X$ therefore depends on the sub-sample (i.e. $X^{\text {Risk }}$ is $100 \times 6, X^{\text {Comp }}$ is $100 \times 5$, and $X^{A l l}$ is $\left.100 \times 11\right)$. Table 1 shows the resulting projection vectors for the first principal component (PC), $P C_{1}$.

One method of analysing the relation between PCs and the underlying sample is to look at fractions of 
sample variance explained by each PC. This is possible, because the sum of PC variances matches the sum of variances of the underlying random variables in a sample (i.e. $\sum_{j=1}^{k} \operatorname{Var}\left[P C_{j}\right]=\sum_{j=1}^{k} s_{x_{j}, x_{j}}$ ). The fraction of variance explained by each $\mathrm{PC}$ can therefore be measured as $\frac{\operatorname{Var}\left[P C_{j}\right]}{\sum_{j=1}^{k} \operatorname{Var}\left[P C_{j}\right]}$. If the fraction of explained variance for the first one or two PCs is very high, we know that the underlying random variables are in fact mainly driven by some latent factors represented by those two PCs. Figure 4 depicts the fractions of sample variance explained by each of the principal components (PCs).

When only considering risk factors, the sample variance appears to be distributed fairly evenly among PCs. If we assume risk to be some latent variable that we expect the risk factors to be proxies of, the finding contradicts this assumption. Instead, the chosen risk factors appear to proxy for various independent latent factors. The opposite is true for the group of complexity factors, where the first PC explains more than 60 percent of the sample variation. All remaining PCs each explain less than 20 percent at the most. This reveals that the chosen complexity factors - at least in large parts track the same underlying latent complexity factor. When including both risk and complexity factors in the PCA, the first PC explains around 40 percent of the sample variation and the next three or four PCs add another 10 to 20 percent each.

In Figure 5 we plot the correlation of each of the risk and complexity factors with the first two $\mathrm{PCs}$ for each of the factor sample subsets. Note that only the absolute correlation value is relevant when interpreting these correlations because PCs are not determined in their sign. Our results support the previous discussion regarding the explained sample variance. While the absolute correlation for risk factors with both $P C_{1}$ and $P C_{2}$ range from zero to 1.0 (top left panel), absolute correlations for complexity factors lie clearly within a range from 0.5 to 1.0 with a strong tendency towards higher values (top right panel). In the bottom left panel we note the absence of a clear correlation pattern between factors and the first two PCs. With the exception of the "number of structural" layers factor all complexity factors maintain a strong correlation with $P C_{1}$. Risk factors deviate very clearly from their correlations with both PCs in the top left panel.

Figure 6, Figure 7, and Figure 8 plot the first three PCs against each other and show the correlation matrix eigenvalues associated with each principle component.

Finally, we plot the expert score of each security in the sample against its first PC in Figure 9. As can be seen there is a clear relation between the total score and the first PC for risk, complexity, and both risk and complexity factors. This relation is most evident for the latter two groups. 

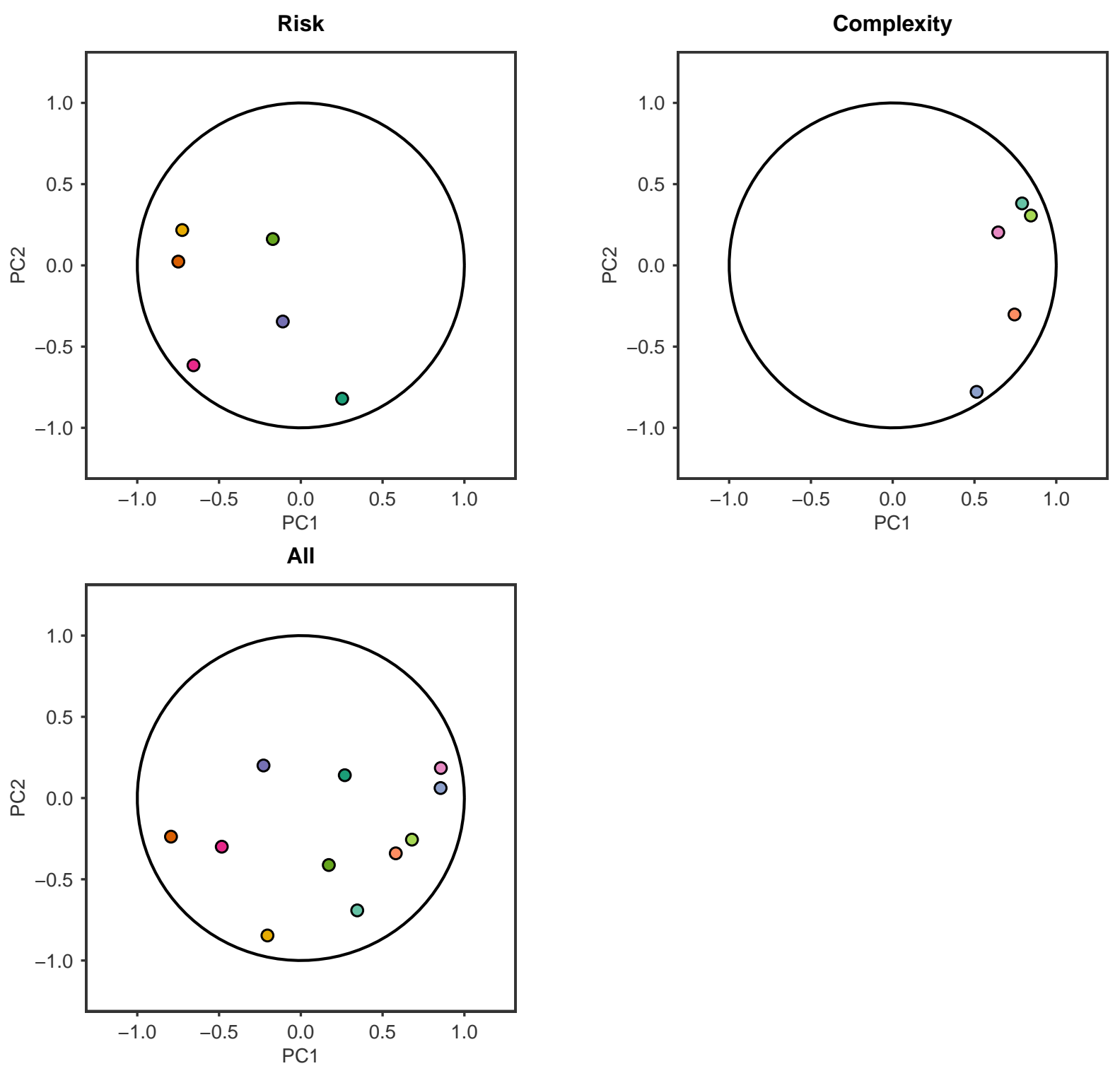

Figure 5: Correlations of the factors with the first two PCs, based on the PCA of only risk, only complexity, and both risk and complexity factors The risk factors are volatility, liquidity, credit rating, duration / cash flow, leverage, and diversification degree. The complexity factors comprise of the number of structural layers, expansiveness of derivatives, availability \& known pricing models, number of return outcome scenarios, and transparency / ease of understanding. 

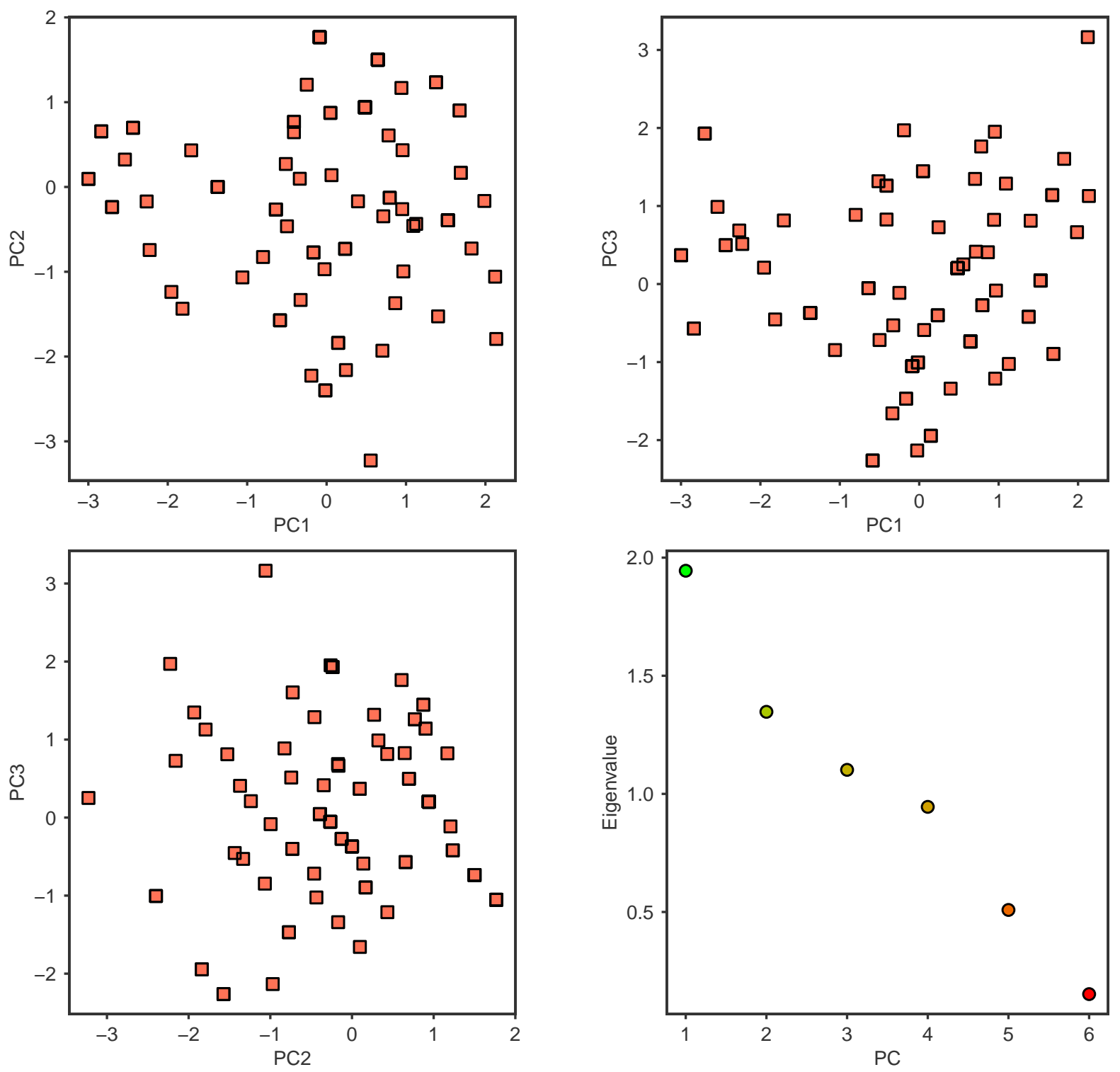

Figure 6: The first three PCs derived from the PCA of the risk factors plotted against each other (top left, top right, and bottom left) and the eigenvalues of the polychoric correlation matrix of risk factors (bottom right). 

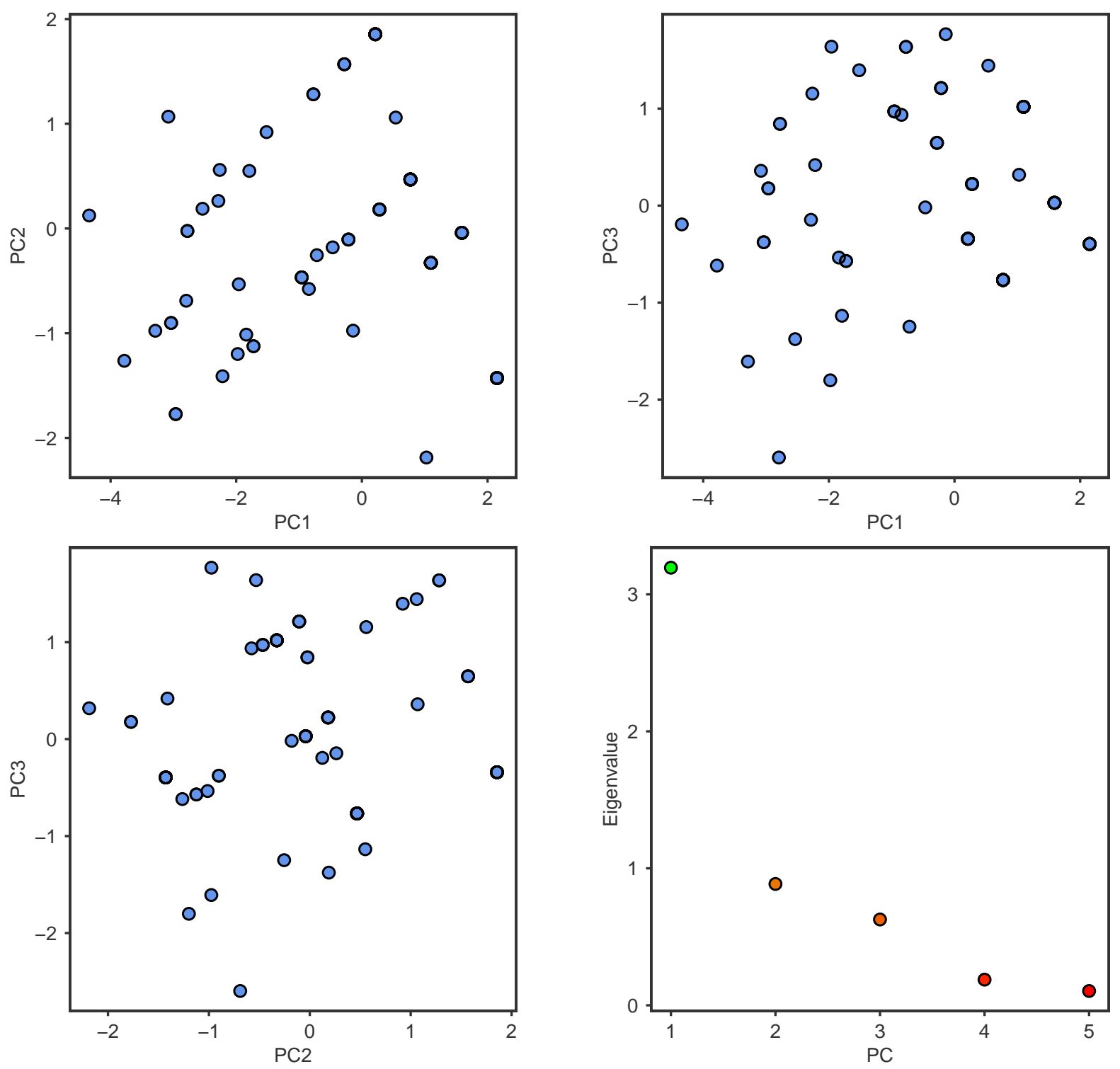

Figure 7: The first three PCs derived from the PCA of the complexity factors plotted against each other (top left, top right, and bottom left) and the eigenvalues of the polychoric correlation matrix of complexity factors (bottom right). 

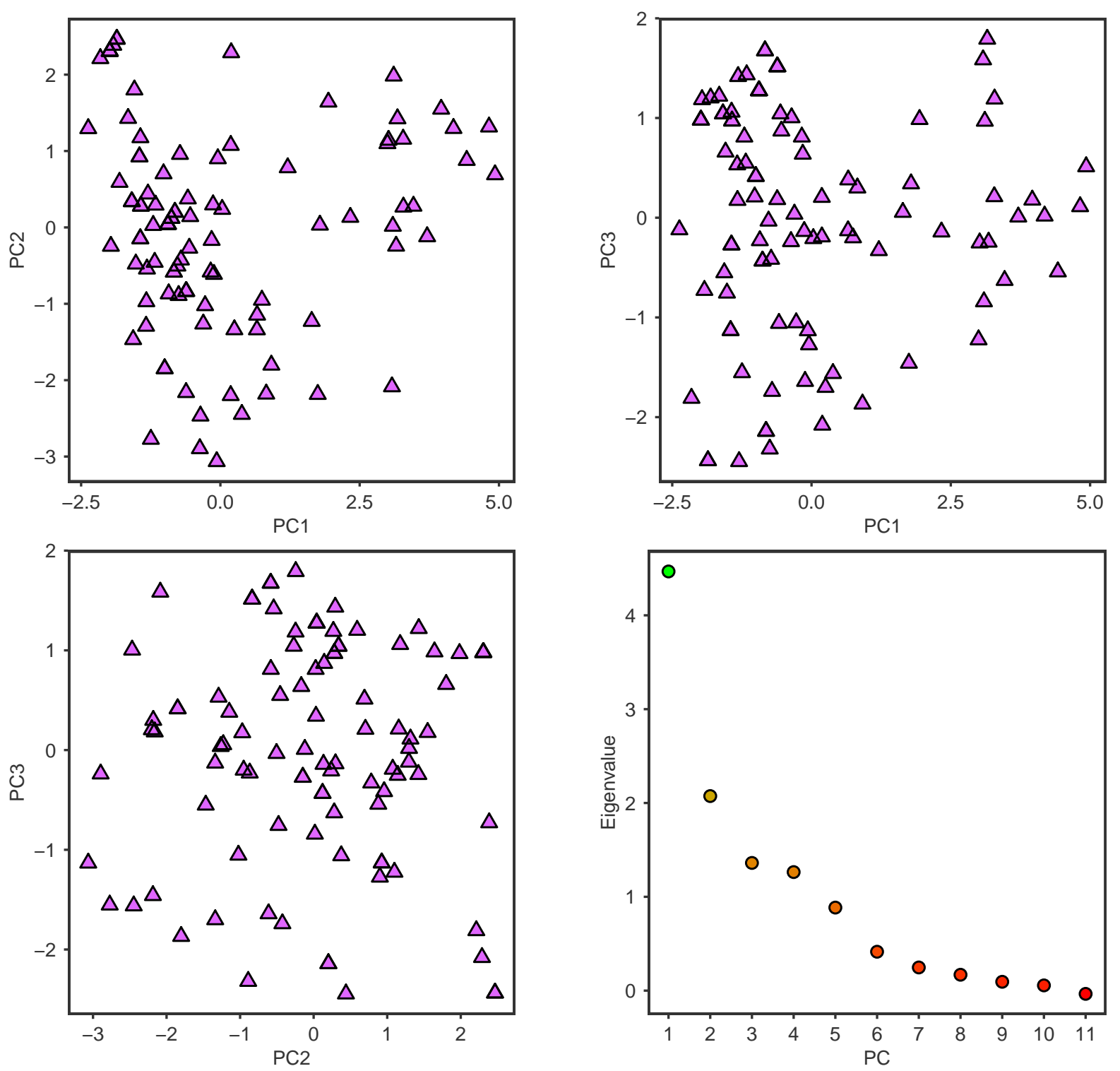

Figure 8: The first three PCs derived from the PCA of the risk and complexity factors plotted against each other (top left, top right, and bottom left) and the eigenvalues of the polychoric correlation matrix of risk and complexity factors (bottom right). 

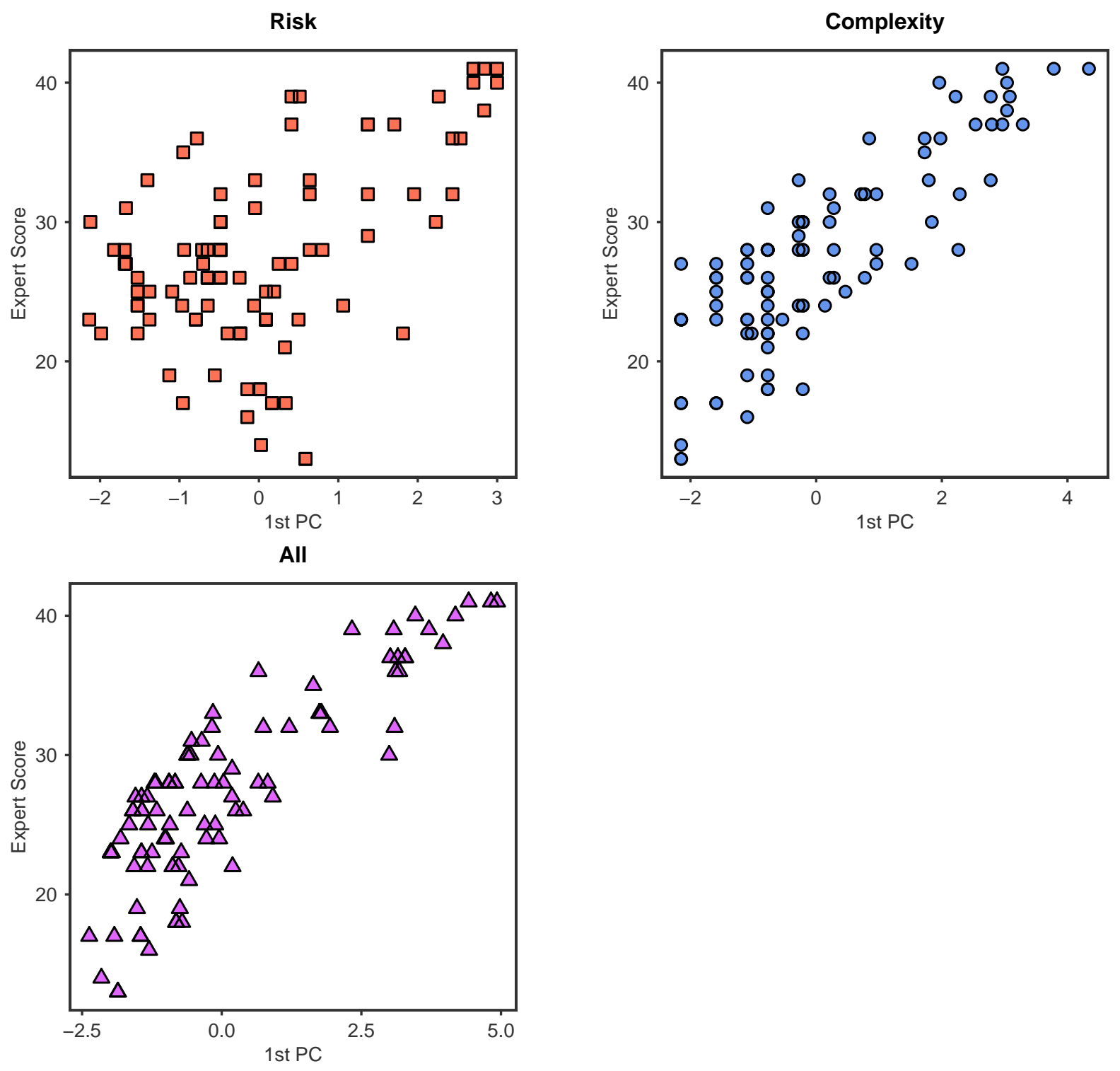

Figure 9: The first PC from the PCA of risk (top left), complexity (top right), and risk and complexity (bottom left) factor scores plotted against the expert score of the corresponding securities. 
Risk

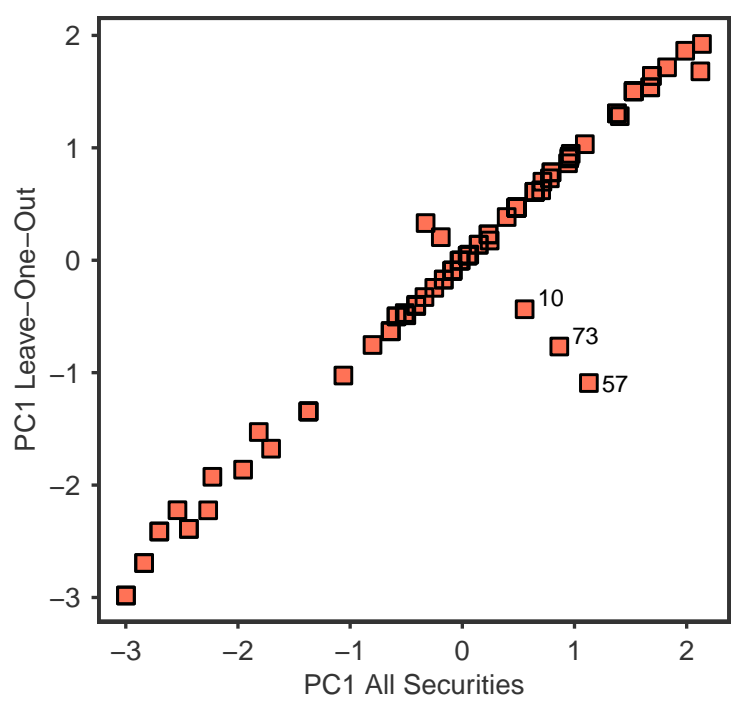

All

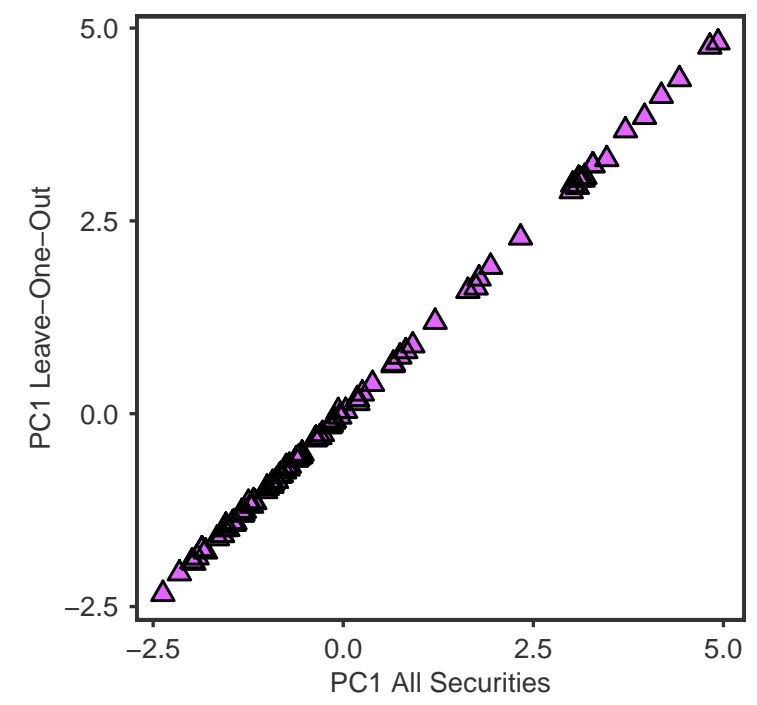

Complexity

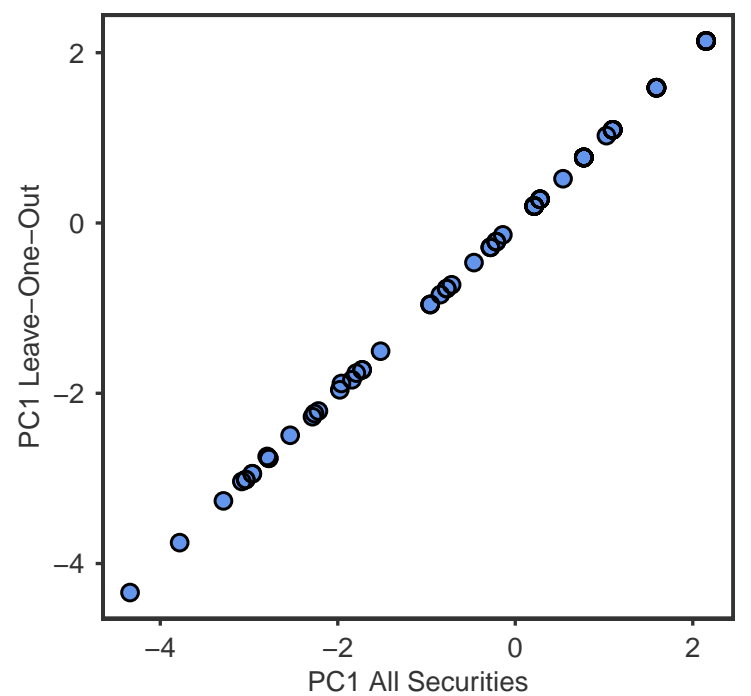

Figure 10: $\hat{f}_{1}\left(x_{i}\right)$ plotted against $f_{1}\left(x_{i}\right)$ for risk factors (top left), for complexity factors (top right), for risk and complexity factors (bottom). 10.0655, 0.0219, and 0.2899 in each setup respectively. Outliers are labeled with their security index in the sample.

\subsection{Cross Validation via Leave-One-Out}

The PCA results are cross validated by employing a leave-one-out (LOO) procedure. We compute the first PC for a security $i$ based on weights obtained from a PCA of the sample excluding security $i$. In Figure 10 we plot the LOO PCs against their regular counterparts. Additionally, we define a function

$$
R_{1}=\sum_{i=1}^{n}\left\{f_{1}\left(x_{i}\right)-\hat{f}_{1}\left(x_{i}\right)\right\}^{2}
$$

where $f_{1}\left(x_{i}\right)$ is the first $\mathrm{PC}$ for security $i$ resulting from a PCA of the whole sample and $\hat{f}_{1}\left(x_{i}\right)$ is the first PC for security $i$ computed from the weights of a PCA of the sample of $n-1$ securities (i.e. excluding security $i$ ). The values of $R_{1}$ for the three samples $X^{\text {Risk }}, X^{\text {Comp }}$, and $X^{A l l}$ are 10.0655 , 0.0219, and 0.2899, respectively. From these results we take that the PCA has some stability issues when only considering risk factors. Otherwise results are stable. 


\section{Adjusted Weighting of Factor Scores}

In the following we consider two different applications of adjusting the weights applied to $X$. First, we try to find a weighting vector $w \in \mathbb{R}^{k}$ such that the projection $x_{i} w$ for each security $i$ is as close as possible to its known expert score. Second, we evaluate the maximum distance between the projections of $X$ through randomly chosen random vectors $w$.

\subsection{Match Expert Score}

Given a matrix $X_{1} \in\{1,2,3\}^{n \times k}, X_{2} \in\{1,3,5\}^{n \times k}$, or $X_{3} \in\{1,4,9\}^{n \times k}$ and again considering the sub-samples $X^{R i s k}, X^{C o m p}$, or $X^{A l l}$, we can compute a function

$$
R_{2}(X, w)=X w-f
$$

where $w$ is an $k \times 1$ vector of weights and $f$ is an $n \times 1$ vector of expert scores. From this we derive two optimisation problems (OPs) $O P_{1}$ and $O P_{2}$,

$$
\widehat{w}_{O P_{1}}=\arg \min _{w_{O P_{1}}}\left\|X w_{O P_{1}}-f\right\|_{1},
$$

and

$$
\widehat{w}_{O P_{2}}=\arg \min _{w_{O P_{2}}}\left\|X w_{O P_{2}}-f\right\|_{2}^{2}
$$

respectively. Table 2 shows the optimal weights for both OPs using one of $X_{1}, X_{2}$, or $X_{3}$ and either risk factors, complexity factors, or both risk and complexity factors. Figures 11 through 16 show the resulting weighted scores $X \widehat{w}$ plotted against the known expert scores.

As can be seen in our results, the linear approximation of expert scores is hard, even when using all 11 factors. The sum of squared approximation errors, $R_{2}^{*}$, in Table 2 is lowest for $X_{1}$ and the use of all factors. A discrete scale of $\{1,2,3\}$ thus appears better suited than the alternatives $\{1,3,5\}$ and $\{1,4,9\}$. 

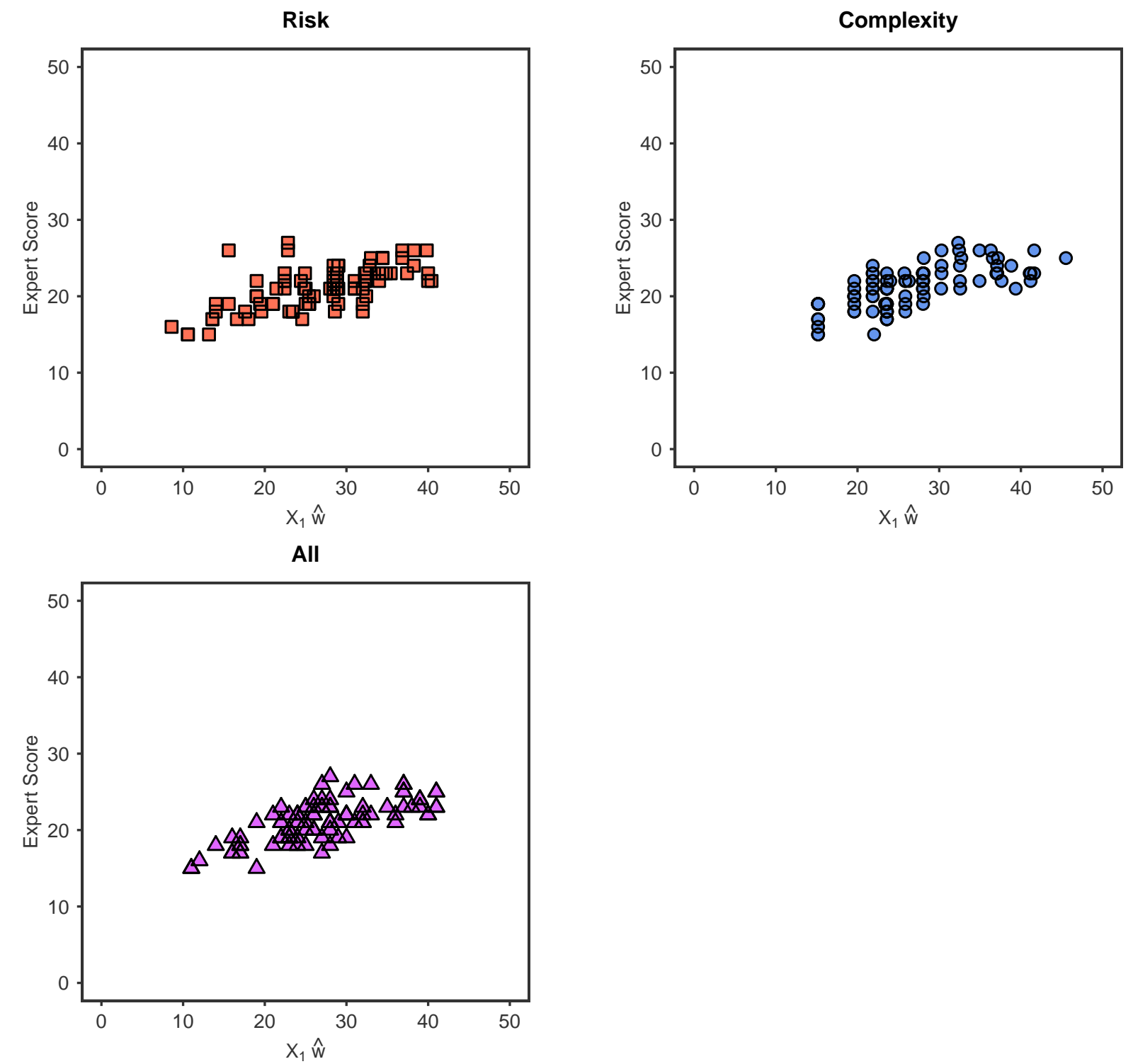

Figure 11: The expert score $(f)$ plotted against $X_{1} \widehat{w}$ for $O P_{1}$. We distinguish between results for risk factors (top left), complexity factors (top right), and risk and complexity factors (bottom left). 


\begin{tabular}{|c|c|c|c|c|c|c|}
\hline \multicolumn{7}{|c|}{ Panel A: Risk factors } \\
\hline & \multicolumn{2}{|c|}{$X_{1}$} & \multicolumn{2}{|c|}{$X_{2}$} & \multicolumn{2}{|c|}{$X_{3}$} \\
\hline & $O P_{1}$ & $O P_{2}$ & $O P_{1}$ & $O P_{2}$ & $O P_{1}$ & $O P_{2}$ \\
\hline$\overline{\widehat{w}_{1}}$ & 0.7773 & 0.7882 & 0.8020 & 0.8123 & 0.6574 & 0.7511 \\
\hline$\widehat{w}_{2}$ & -0.2064 & -0.1632 & -0.1433 & -0.0702 & 0.0750 & 0.0529 \\
\hline$\widehat{w}_{3}$ & 0.0346 & 0.0289 & 0.1299 & 0.1182 & 0.1069 & 0.0946 \\
\hline$\widehat{w}_{4}$ & -0.1650 & -0.1170 & -0.1433 & -0.1328 & -0.1737 & -0.1412 \\
\hline$\widehat{w}_{5}$ & 0.4952 & 0.5499 & 0.4596 & 0.5077 & 0.6352 & 0.5829 \\
\hline$\widehat{w}_{6}$ & 0.2821 & 0.1876 & 0.2962 & 0.2142 & 0.3424 & 0.2536 \\
\hline$\rho_{A \widehat{w}, f}$ & 0.7729 & 0.8296 & 0.7562 & 0.7920 & 0.6380 & 0.7664 \\
\hline$R_{2}^{*}$ & 334.8355 & 1845.7312 & 408.8966 & 2698.5647 & 487.7317 & 3924.8506 \\
\hline
\end{tabular}

Panel B: Complexity factors

\begin{tabular}{lrrrrrr}
\hline \hline & \multicolumn{2}{c}{$X_{1}$} & \multicolumn{2}{c}{$X_{2}$} & & $X_{3}$ \\
\cline { 2 - 7 } & $O P_{1}$ & $O P_{2}$ & $O P_{1}$ & $O P_{2}$ & $O P_{1}$ & $O P_{2}$ \\
\hline$\widehat{w}_{1}$ & 0.5636 & 0.5290 & 0.6732 & 0.6777 & 0.5420 & 0.6216 \\
$\widehat{w}_{2}$ & 0.2873 & 0.3255 & 0.2891 & 0.2960 & 0.3391 & 0.2848 \\
$\widehat{w}_{3}$ & -0.0136 & 0.1554 & -0.0008 & 0.0916 & 0.0947 & 0.0498 \\
$\widehat{w}_{4}$ & 0.5849 & 0.5154 & 0.4811 & 0.3873 & 0.1521 & 0.2456 \\
$\widehat{w}_{5}$ & 0.5075 & 0.5695 & 0.4814 & 0.5429 & 0.7477 & 0.6854 \\
\hline$\rho_{A}, f$ & 0.9825 & 0.9924 & 0.9745 & 0.9755 & 0.9535 & 0.9515 \\
$R_{2}^{*}$ & 339.0912 & 1755.0434 & 453.0134 & 3363.2401 & 677.2657 & 6798.8500 \\
\hline \hline
\end{tabular}

Panel C: Risk and complexity factors

\begin{tabular}{lrrrrrr}
\hline \hline & \multicolumn{2}{c}{$X_{1}$} & \multicolumn{2}{c}{$X_{2}$} & & $X_{3}$ \\
\cline { 2 - 7 } & $O P_{1}$ & $O P_{2}$ & $O P_{1}$ & $O P_{2}$ & $O P_{1}$ & $O P_{2}$ \\
\hline$\widehat{w}_{1}$ & 0.6622 & 0.6279 & 0.5914 & 0.6130 & 0.3821 & 0.5261 \\
$\widehat{w}_{2}$ & -0.0003 & -0.0284 & 0.2187 & 0.1362 & 0.4382 & 0.2817 \\
$\widehat{w}_{3}$ & -0.2645 & -0.2019 & -0.1590 & -0.0705 & -0.1454 & -0.0126 \\
$\widehat{w}_{4}$ & 0.1326 & 0.1535 & 0.1182 & 0.1511 & 0.0754 & 0.1186 \\
$\widehat{w}_{5}$ & 0.2651 & 0.3006 & 0.2368 & 0.2868 & 0.2879 & 0.3930 \\
$\widehat{w}_{6}$ & -0.1326 & -0.1486 & -0.1183 & -0.1187 & -0.0669 & -0.0568 \\
$\widehat{w}_{7}$ & 0.2652 & 0.2960 & 0.2368 & 0.2921 & 0.1633 & 0.2432 \\
$\widehat{w}_{8}$ & 0.2650 & 0.2776 & 0.2365 & 0.2566 & 0.1853 & 0.2278 \\
$\widehat{w}_{9}$ & 0.1329 & 0.1695 & 0.1950 & 0.2079 & 0.1391 & 0.2617 \\
$\widehat{w}_{10}$ & 0.3969 & 0.3551 & 0.4945 & 0.3880 & 0.6406 & 0.3581 \\
$\widehat{w}_{11}$ & 0.2653 & 0.3296 & 0.3139 & 0.3695 & 0.2390 & 0.4052 \\
\hline$\rho_{A} \widehat{w}, f$ & 0.9942 & 0.9952 & 0.9768 & 0.9792 & 0.9117 & 0.9513 \\
$R_{2}^{*}$ & 38.0562 & 46.5552 & 91.9700 & 225.6082 & 147.5615 & 589.0314 \\
\hline \hline
\end{tabular}

Table 2: Match Expert Score Weights

Optimal (normalised) weights $\widehat{w} \in \mathbb{R}^{k}$, correlations between $A \widehat{w}$ and $f$, as well as the optimal target function value $R_{2}^{*}$ (this is the actual target function and not $R_{2}$ itself) for $O P_{1}$ and $O P_{2}$ using matrices $X_{1} \in\{1,2,3\}^{k \times n}, X_{2} \in\{1,3,5\}^{k \times n}$, and $X_{3} \in\{1,4,9\}^{k \times n}$. The weights have been normalised to unit vectors to facilitate a comparison with PCA weights and simulation weights. 

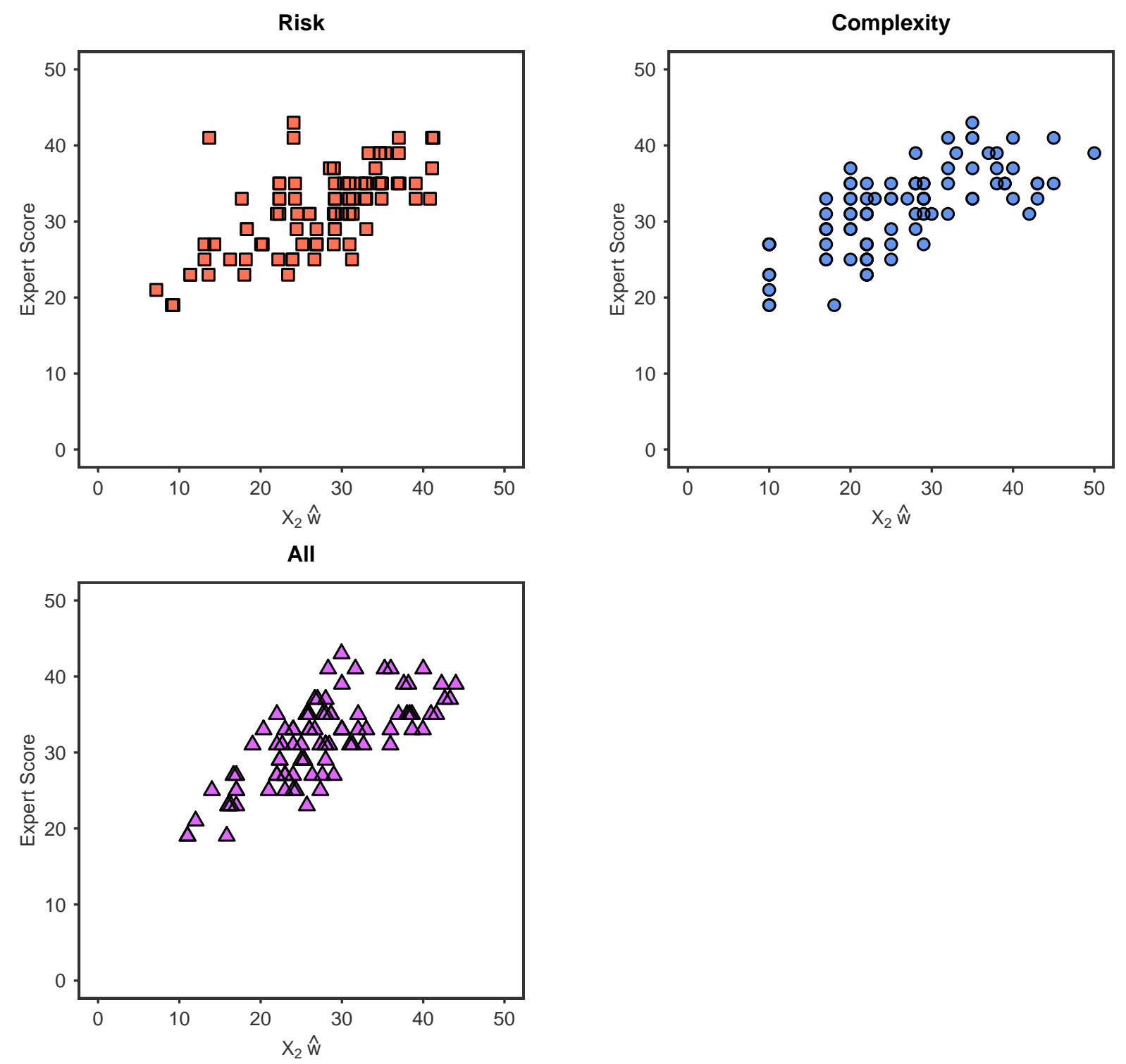

Figure 12: The expert score $(f)$ plotted against $X_{2} \widehat{w}$ for $O P_{1}$. We distinguish between results for risk factors (top left), complexity factors (top right), and risk and complexity factors (bottom left). 

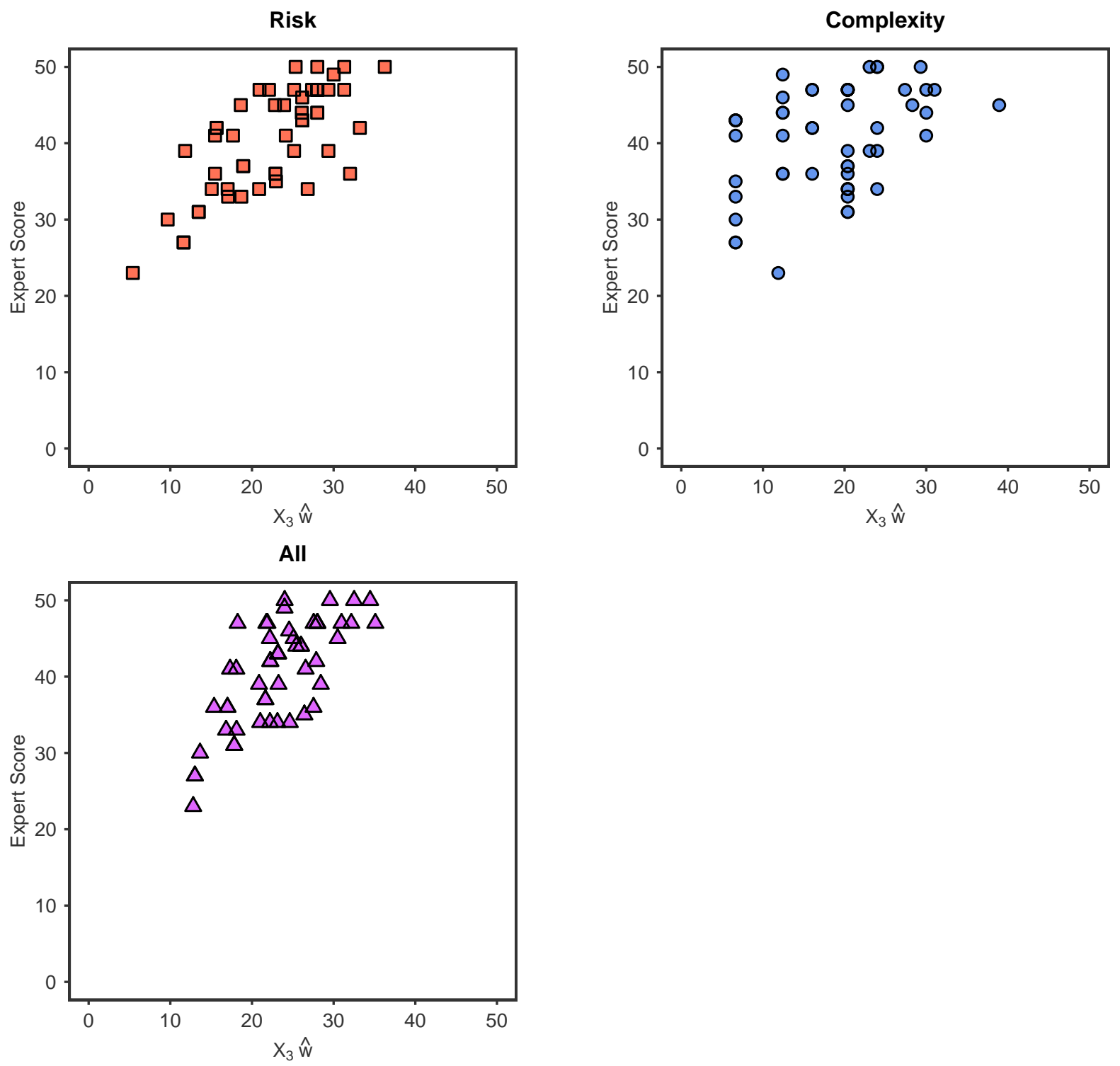

Figure 13: The expert score $(f)$ plotted against $X_{3} \widehat{w}$ for $O P_{1}$. We distinguish between results for risk factors (top left), complexity factors (top right), and risk and complexity factors (bottom left). 

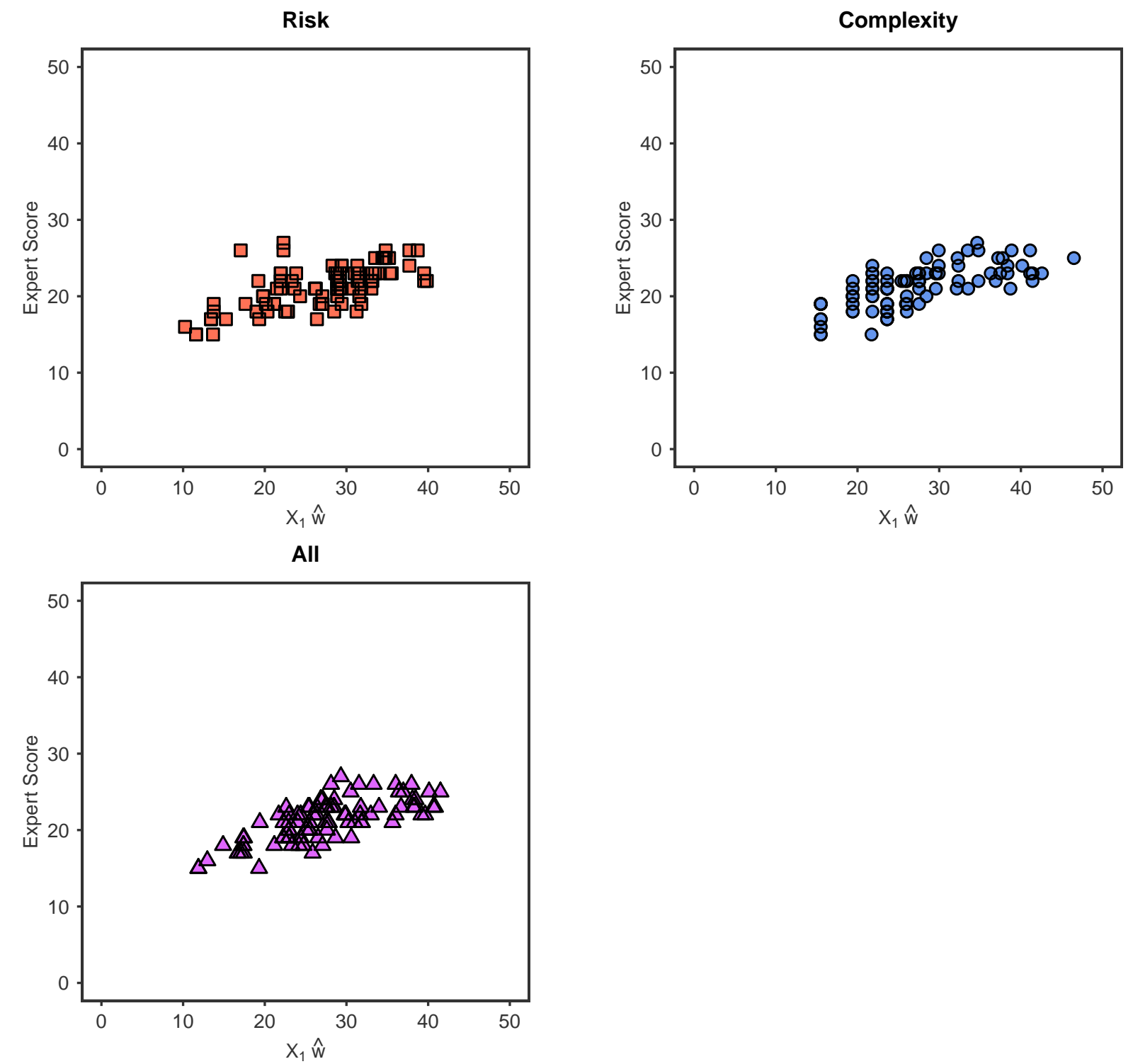

Figure 14: The expert score $(f)$ plotted against $X_{1} \widehat{w}$ for $O P_{2}$. We distinguish between results for risk factors (top left), complexity factors (top right), and risk and complexity factors (bottom left). 

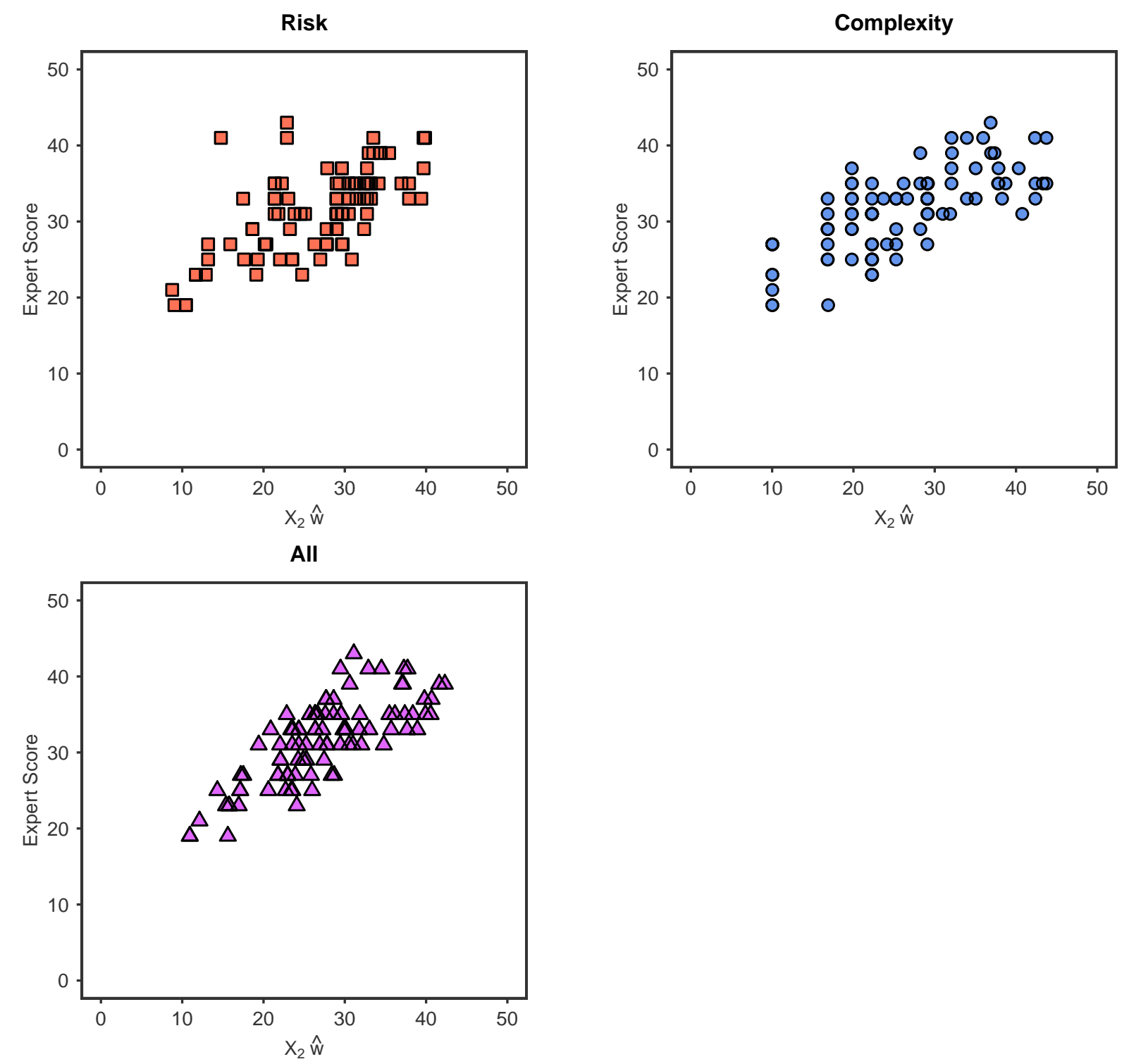

Figure 15: The expert score $(f)$ plotted against $X_{2} \widehat{w}$ for $O P_{2}$. We distinguish between results for risk factors (top left), complexity factors (top right), and risk and complexity factors (bottom left). 

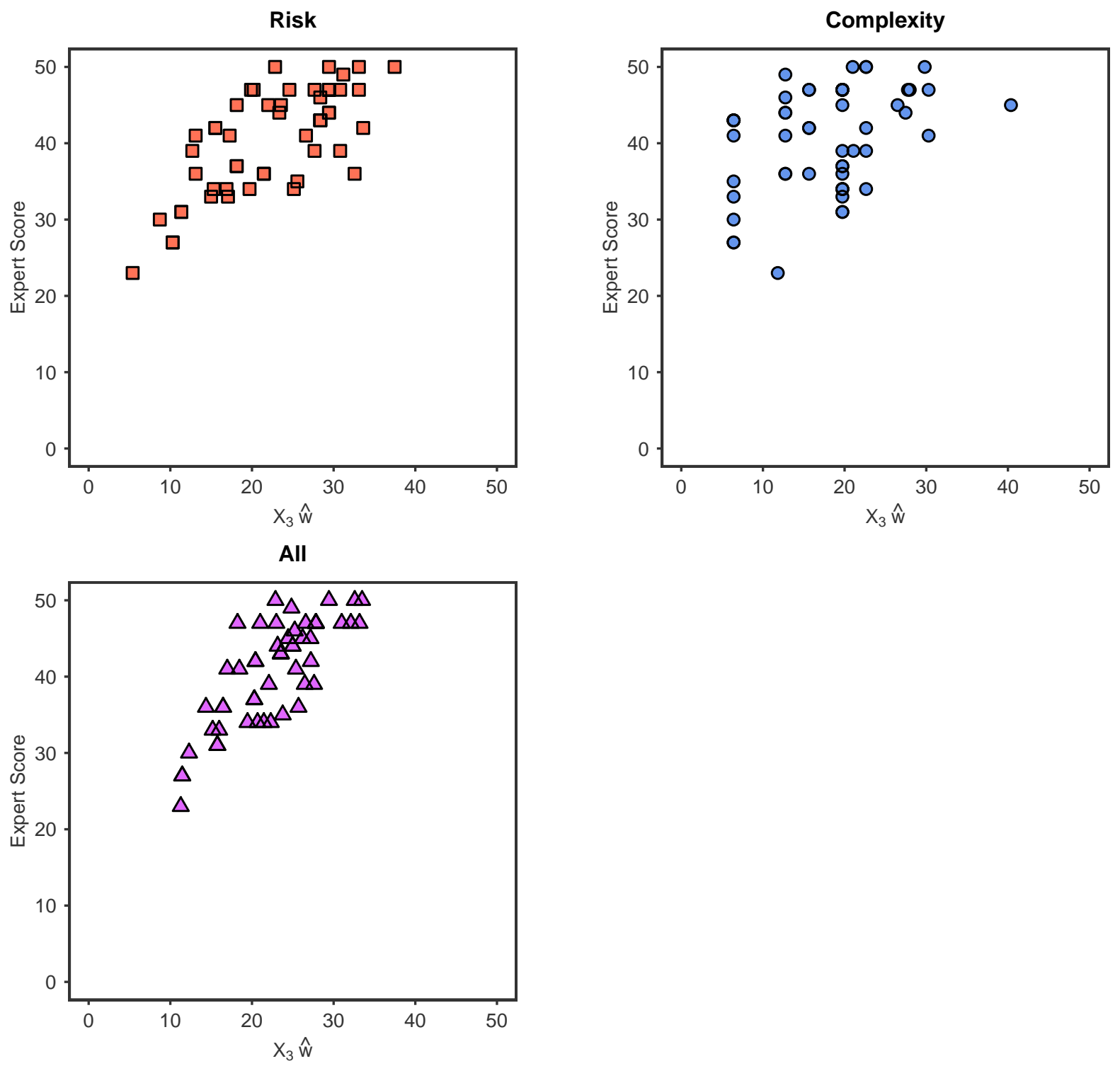

Figure 16: The expert score $(f)$ plotted against $X_{3} \widehat{w}$ for $O P_{2}$. We distinguish between results for risk factors (top left), complexity factors (top right), and risk and complexity factors (bottom left). 

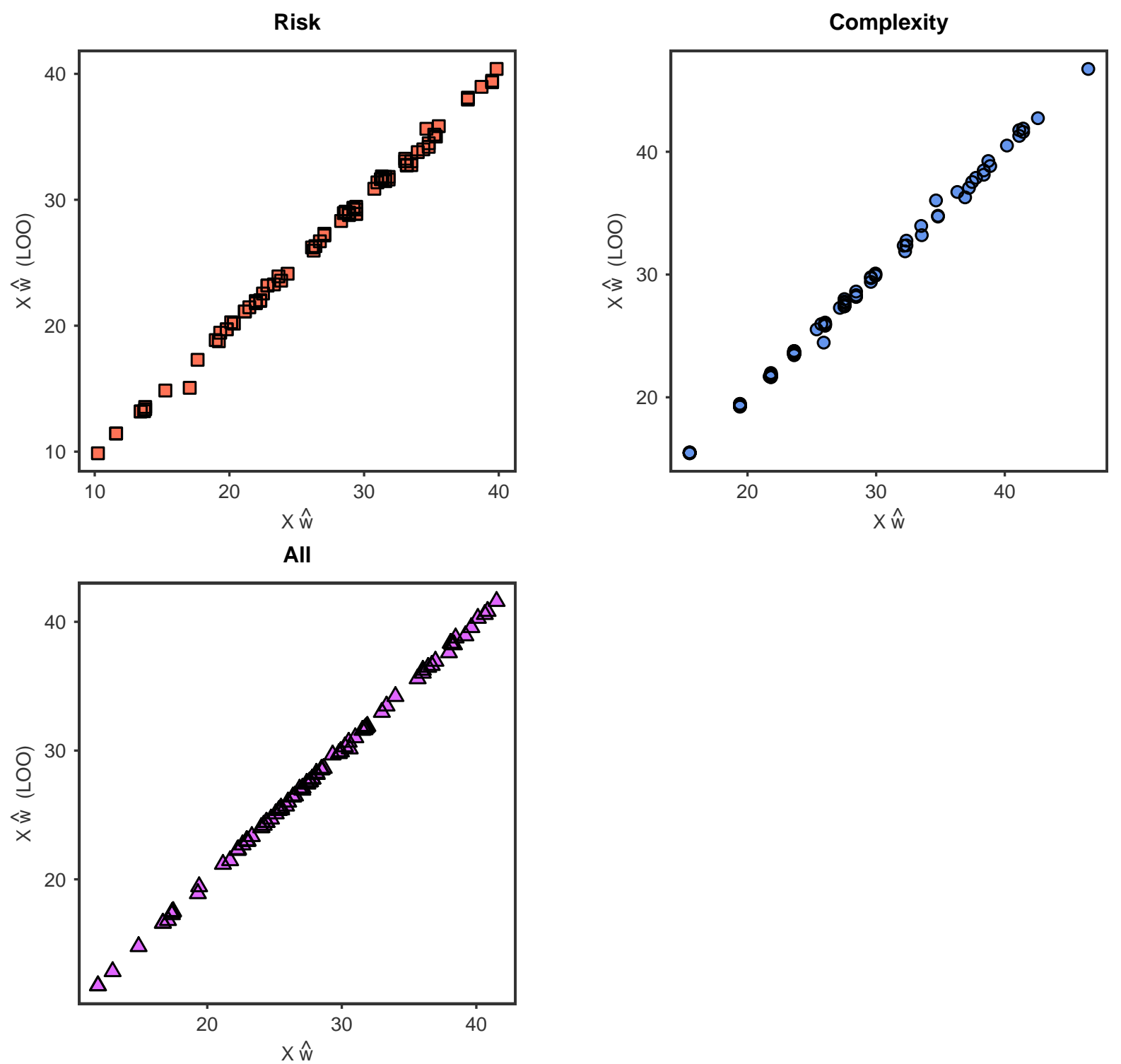

Figure 17: $X_{1} \widehat{w}_{L O O}$ plotted against $X_{1} \widehat{w}$ for $O P_{2}$. We distinguish between results for risk factors (top left), complexity factors (top right), and risk and complexity factors (bottom left).

\subsection{Cross Validation via Leave-One-Out}

As with the PCA, we perform a LOO analysis to see how strongly the optimisation results for (4) depend on individual securities. We only consider $O P_{2}$ for $X_{1}$ because the overall results are best in this specification. The results, depicted in Figure 17, are fairly robust against sample modifications. This is particularly true for $X_{1}^{A l l}$. 


\begin{tabular}{c|rrr}
\hline \hline & $X^{\text {Risk }}$ & $X^{\text {Comp }}$ & $X^{\text {All }}$ \\
\hline$w_{1}$ & -0.9275 & 0.5161 & 0.4319 \\
$w_{2}$ & 0.1824 & 0.4141 & 0.0833 \\
$w_{3}$ & -0.2483 & 0.3300 & 0.0257 \\
$w_{4}$ & 0.1164 & -0.3030 & -0.2453 \\
$w_{5}$ & 0.0130 & 0.6013 & -0.3244 \\
$w_{6}$ & 0.1764 & & -0.2807 \\
$w_{7}$ & & & -0.1371 \\
$w_{8}$ & & & -0.3083 \\
$w_{9}$ & & & 0.1572 \\
$w_{10}$ & & & -0.5835 \\
$w_{11}$ & & & -0.2874 \\
\hline \hline
\end{tabular}

Table 3: Top Ten Mean Maximum Spread Simulation Weights

The mean of the weighting vectors projecting the ten largest spreads from the original score matrix. The mean vectors for $X^{\text {Risk }}, X^{C o m p}$, and $X^{A l l}$ are normalised to unit vectors.

\subsection{Widest Projection Spread}

Given some random $k \times 1$ weighting vector we can compute the maximum spread between each projection in $X w$ and its nearest neighbour. We define $z=X w$ and then consider the order statistics of the elements $z_{i}$ of $z$ (i.e. $\forall i=1, \ldots, n-1: z_{(i)} \leq z_{(i+1)}$ ). The maximum spread between all $z_{(i)}$ and their respective nearest neighbour is then given by

$$
R_{3}(z)=\max _{i}^{n-1}\left(z_{(i+1)}-z_{(i)}\right) .
$$

To examine the influence of the weighting vector $w$ on the maximum projection spread we generate $1000 k \times 1$ uniform random vectors $\left(w \sim \mathcal{U}(-1,1)^{k}\right)$. These vectors are then scaled to unit vectors.

Figure 18 shows the resulting 1000 simulated maximum spreads. The mean maximum spreads for the risk, complexity, and both risk and complexity cases are $\bar{s}^{\text {Risk }}=0.6807, \bar{s}^{\text {Compl }}=0.74725$, $\bar{s}^{A l l}=0.6904$. A box plot of the results is shown in Figure 19. 

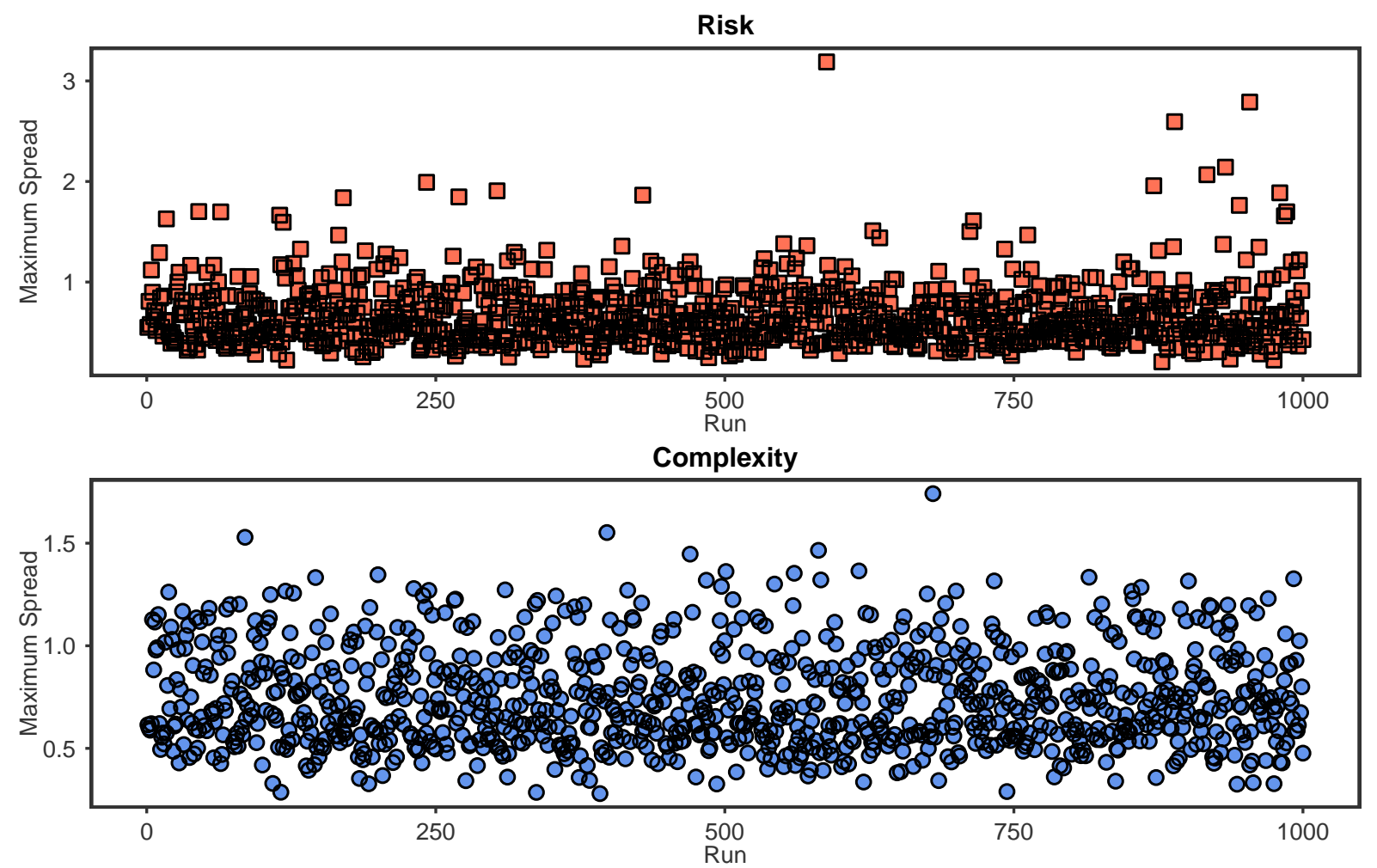

All

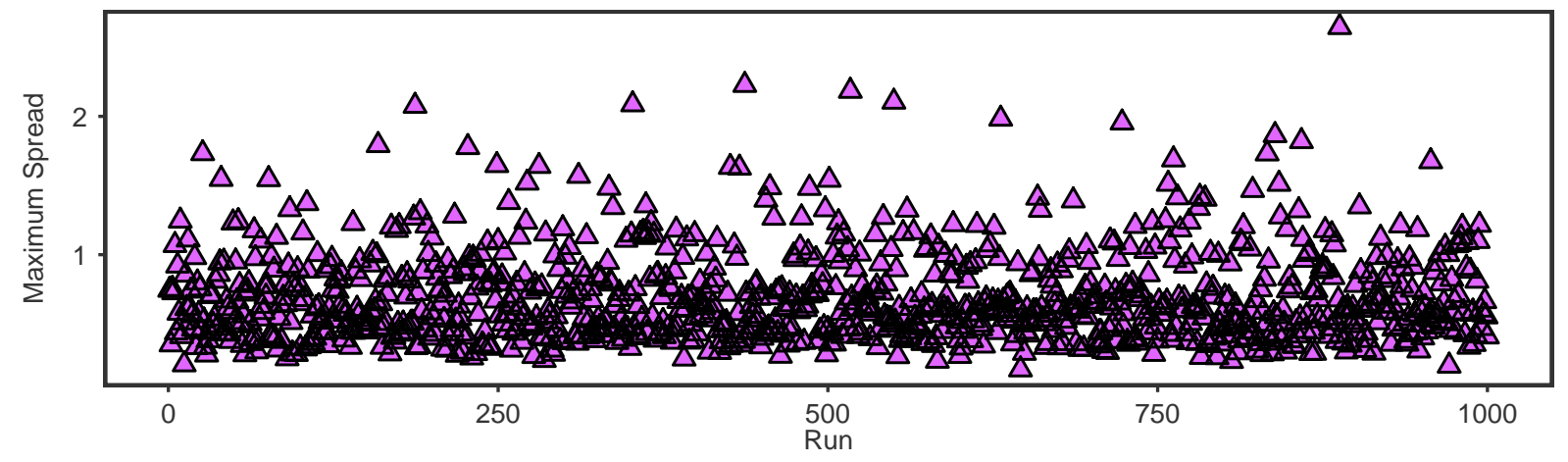

Figure 18: Maximum spread among projections $\mathbf{X}_{\mathbf{1}} \mathbf{w}$ for 1000 randomly chosen $\mathbf{w}$. 


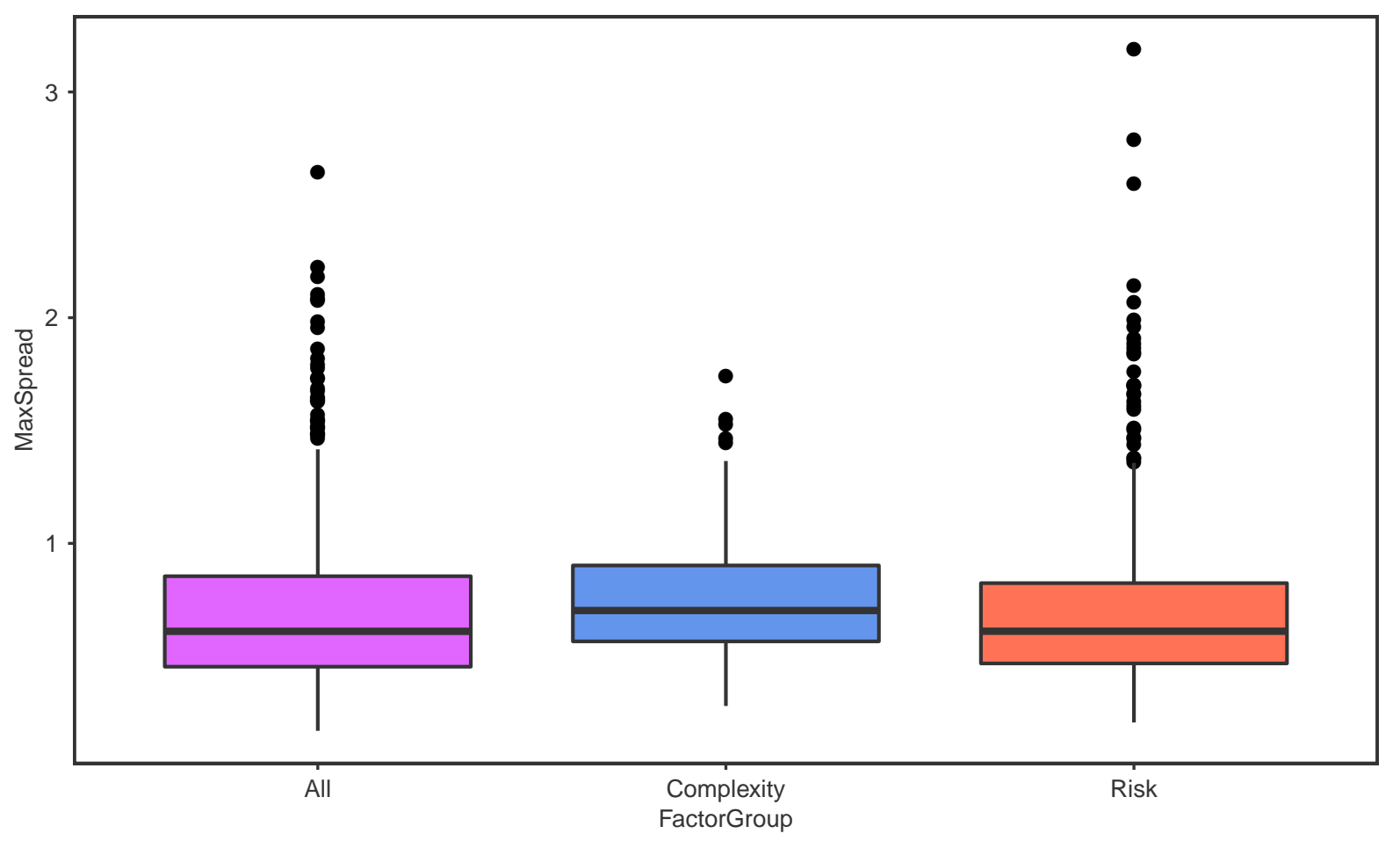

Figure 19: Box plot of maximum spreads among projections $\mathbf{X}_{\mathbf{1}} \mathbf{w}$ for 1000 randomly chosen $\mathbf{w}$. 


\section{Conclusion}

We can summarise our results in a few key points:

1. The choice of risk factors, as the PCA has revealed, does not seem to proxy for a single latent source of risk. The opposite is true for the choice of complexity factors.

2. Overall there is a clear positive relation between the first PC of the full PCA, involving all factors, and the expert score of a security as shown in Figure 9.

3. Approximation of the total expert scores through linear projection of the score matrix is possible, but not perfect. We obtain best results by using a score scale of $\{1,2,3\}$ and applying the $L^{2}$ norm during optimisation. 


\section{A Appendix}

\section{PCA Results From Pearson Correlation Matrix}

Risk

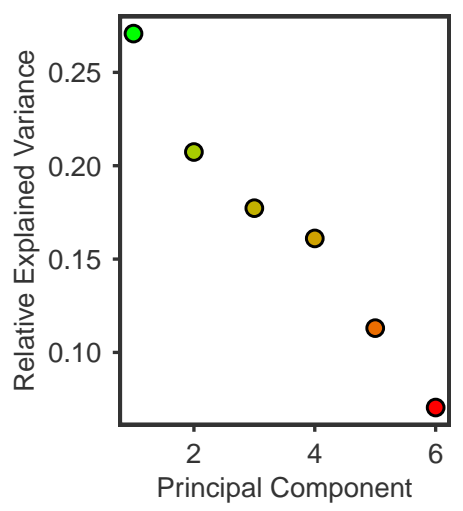

Complexity

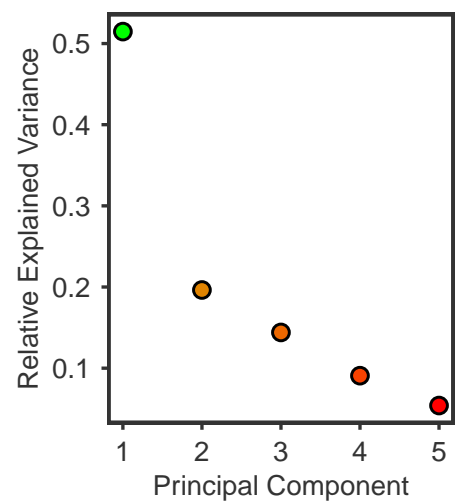

All

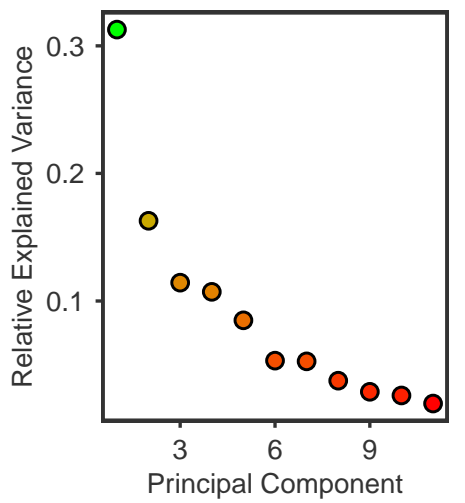

Figure A1: Fraction of variance explained by each of the principal components. 

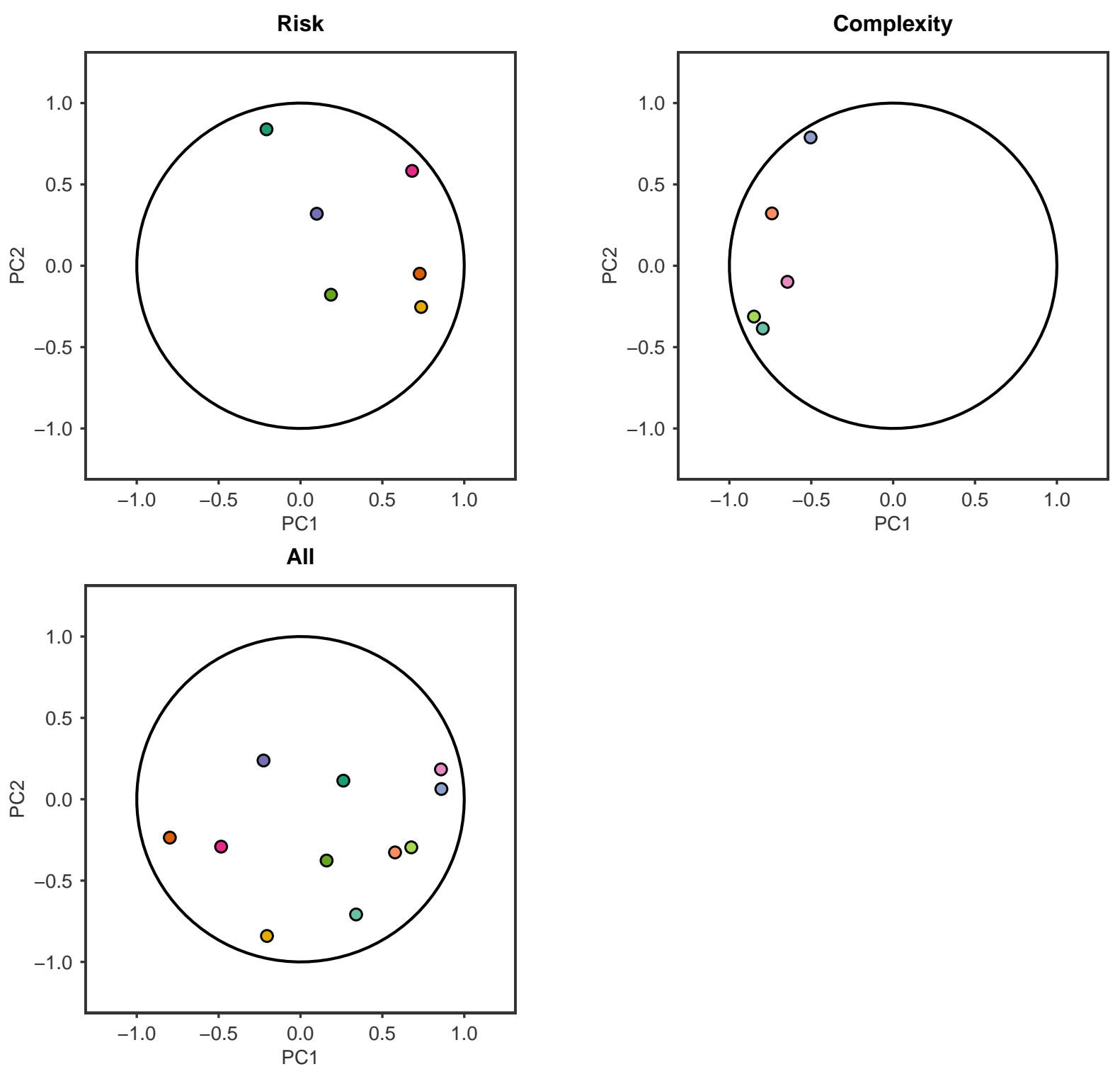

Figure A2: Correlations of the factors with the first two PCs, based on the PCA of only risk, only complexity, and both risk and complexity factors. The risk factors are volatility, liquidity, credit rating, duration / cash flow, leverage, and diversification degree. The complexity factors comprise of the number of structural layers, expansiveness of derivatives, availability \& known pricing models, number of return outcome scenarios, and transparency / ease of understanding. 

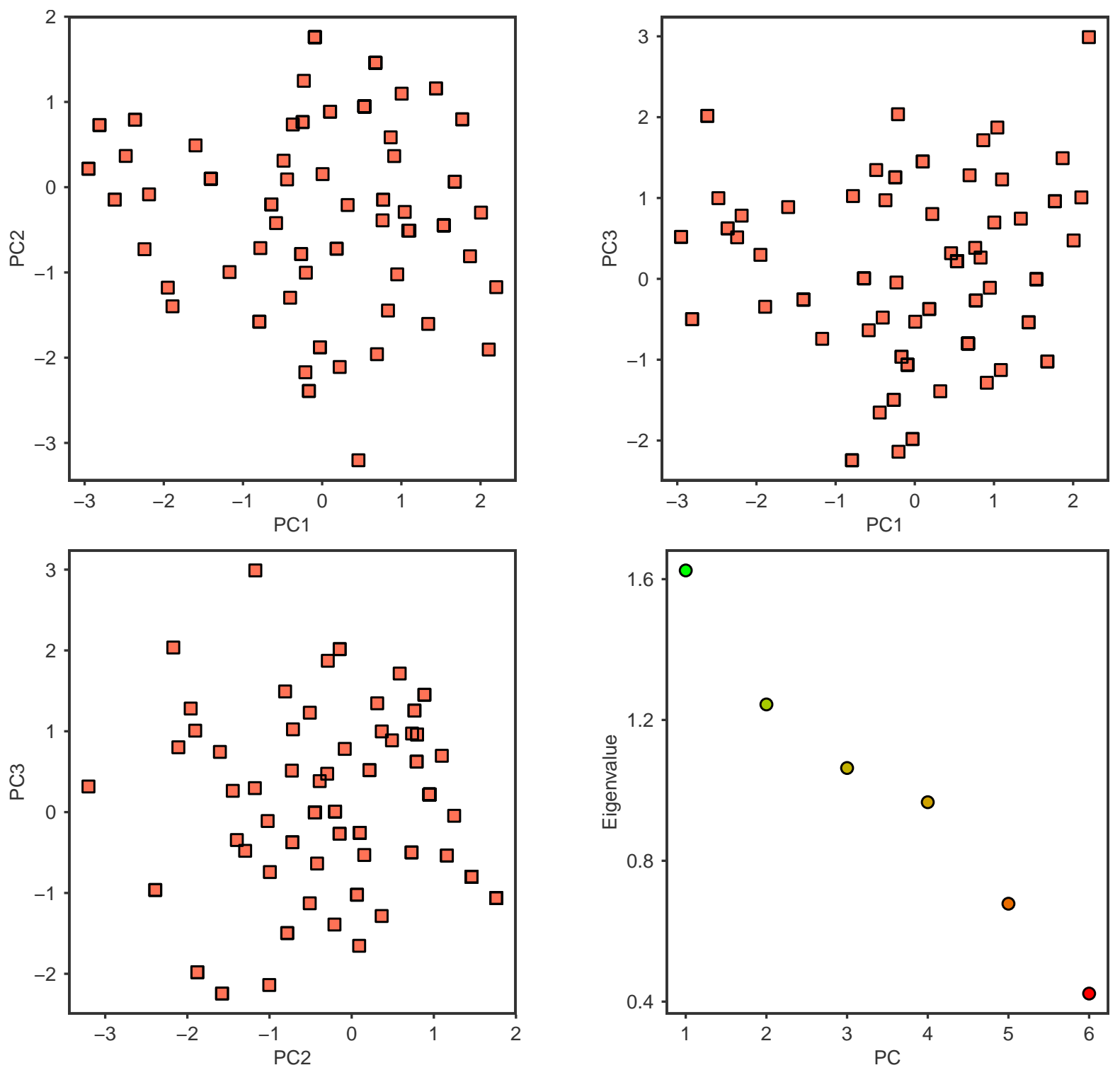

Figure A3: The first three PCs derived from the PCA of the risk factors plotted against each other (top left, top right, and bottom left) and the eigenvalues of the Pearson correlation matrix of risk factors (bottom right). 

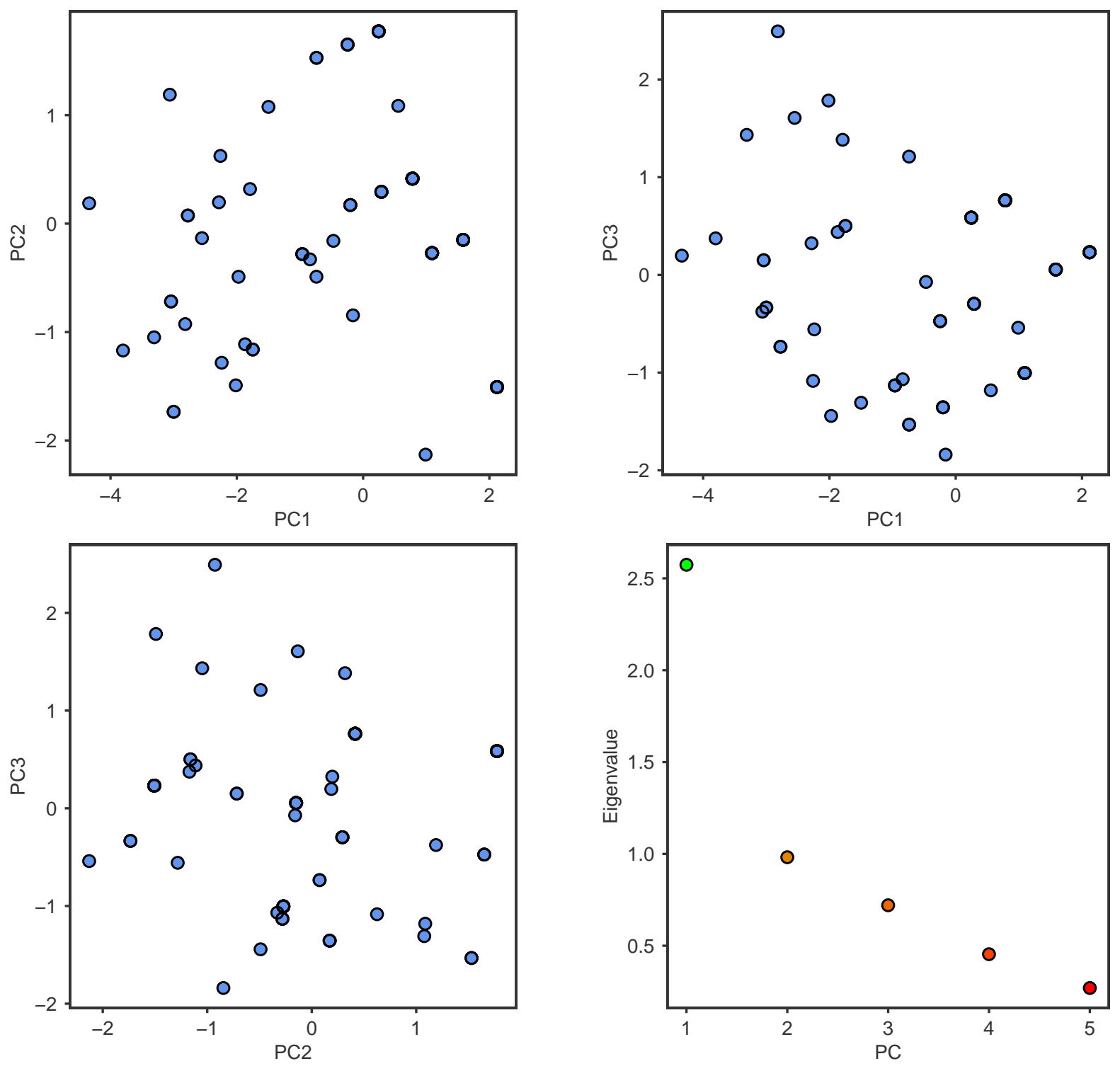

Figure A4: The first three PCs derived from the PCA of the complexity factors plotted against each other (top left, top right, and bottom left) and the eigenvalues of the Pearson correlation matrix of complexity factors (bottom right). 

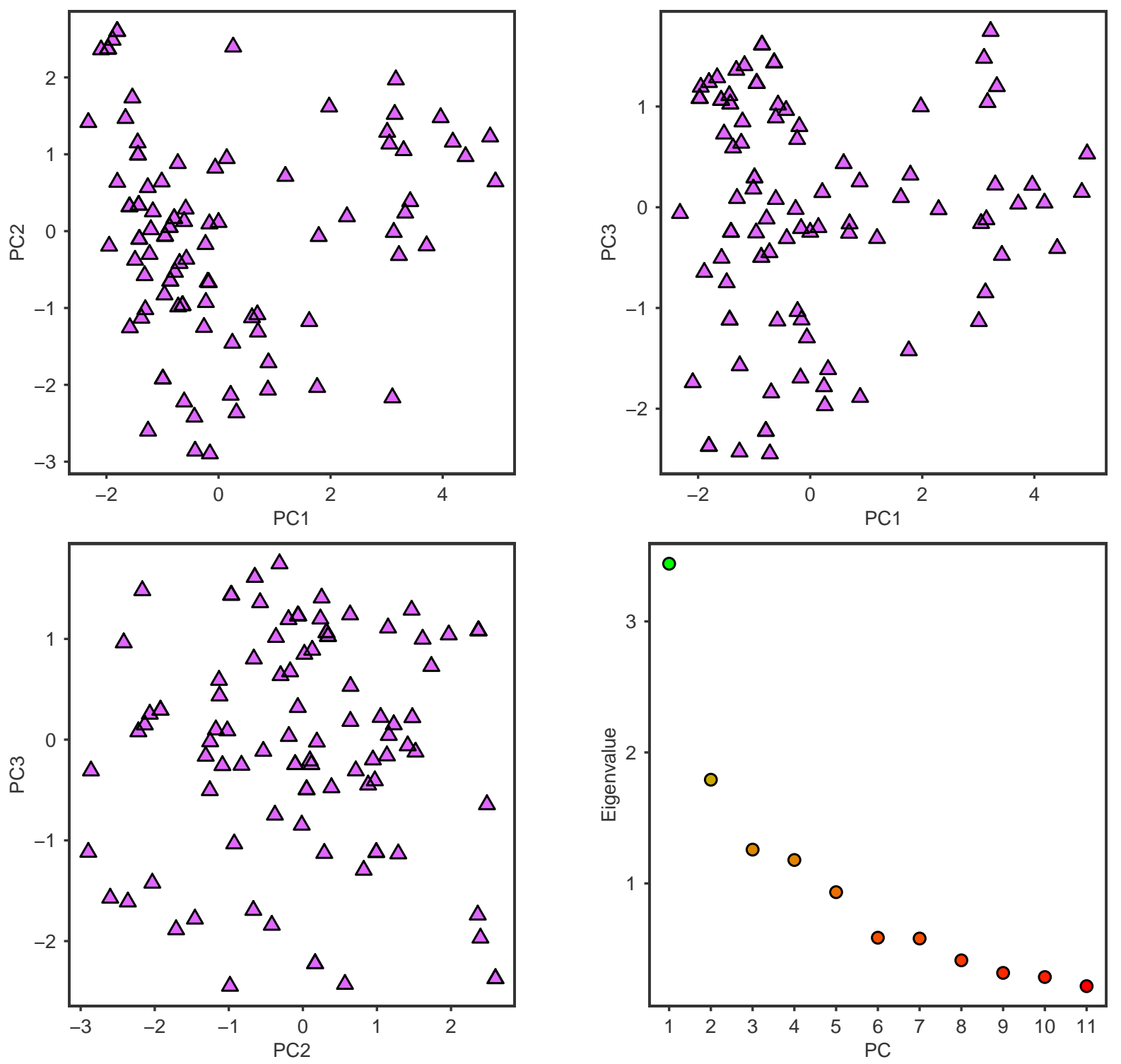

Figure A5: The first three PCs derived from the PCA of the risk and complexity factors plotted against each other (top left, top right, and bottom left) and the eigenvalues of the Pearson correlation matrix of risk and complexity factors (bottom right). 
Risk

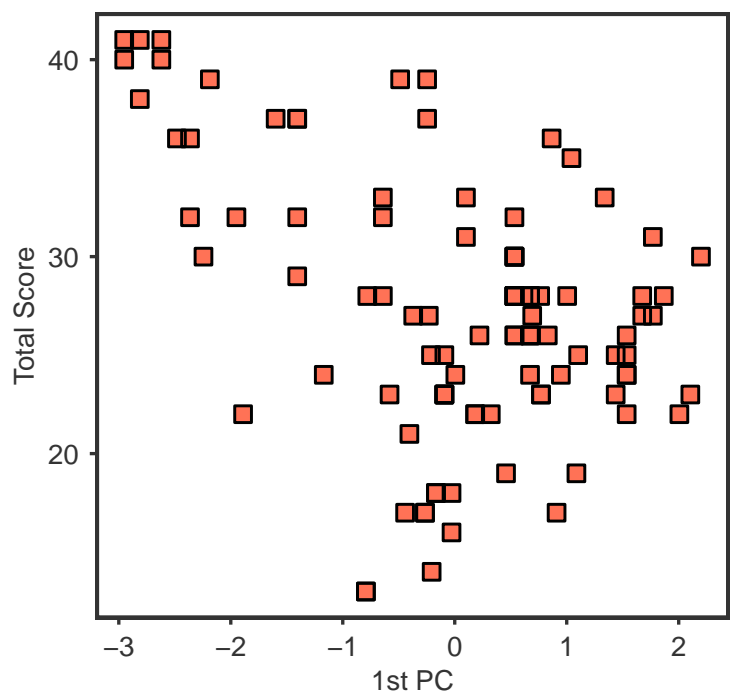

All

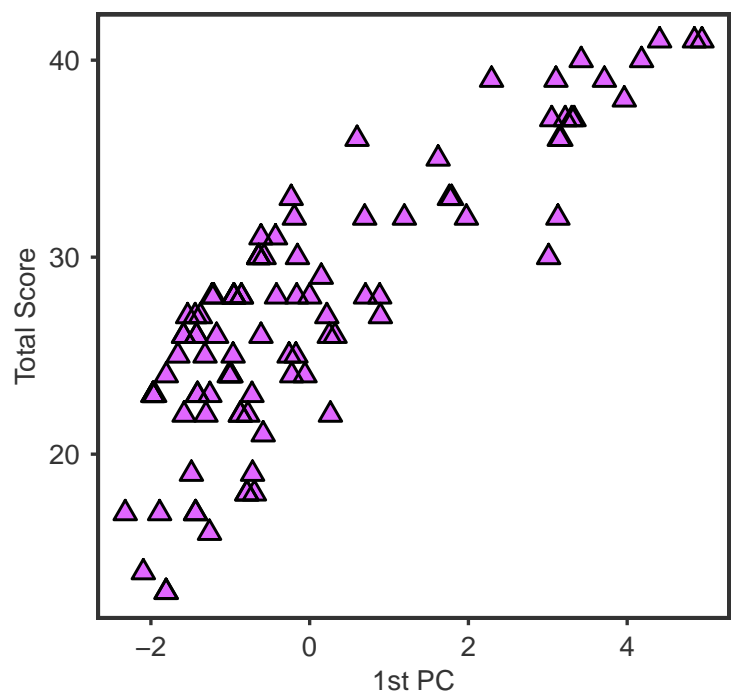

Complexity

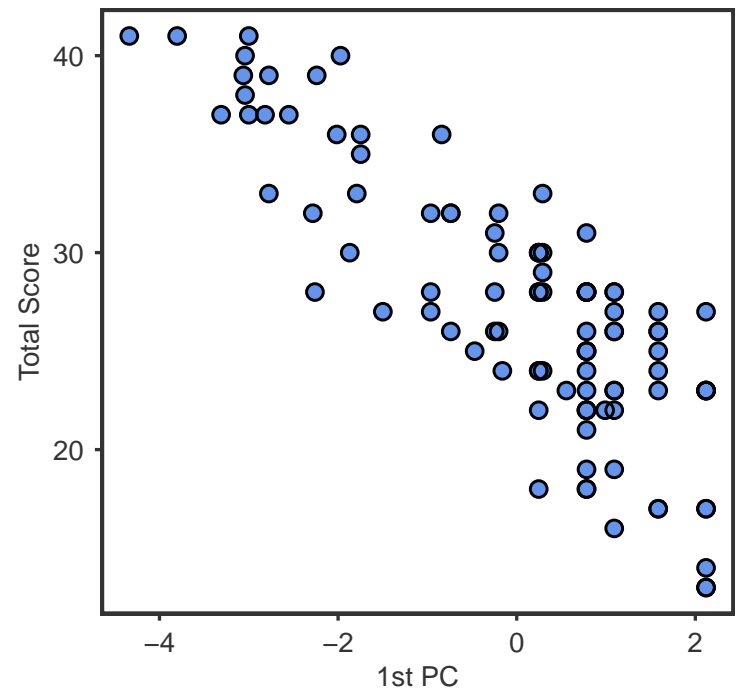

Figure A6: The first PC from the PCA of risk (top left), complexity (top right), and risk and complexity (bottom left) factor scores plotted against the expert score of the corresponding securities. 
Cross Validation via Leave-One-Out

Risk

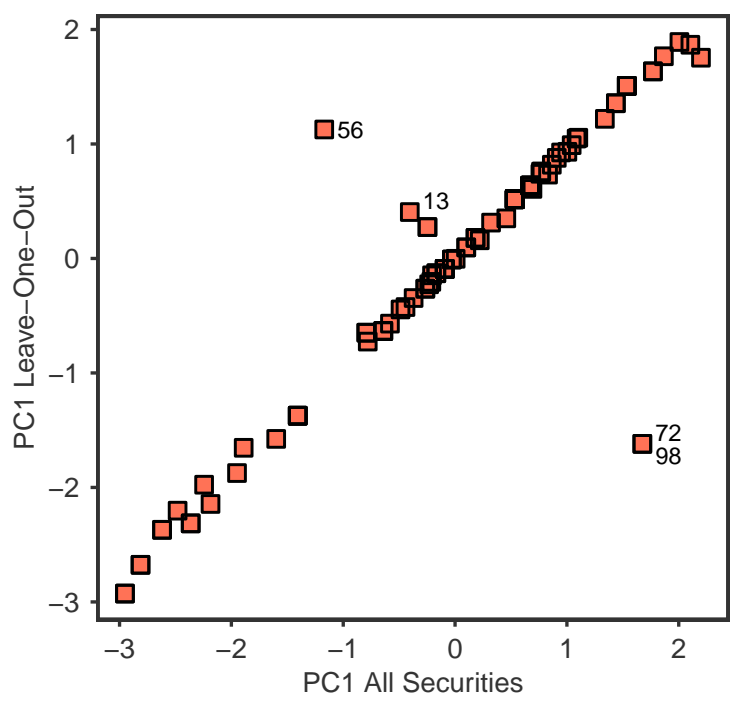

All

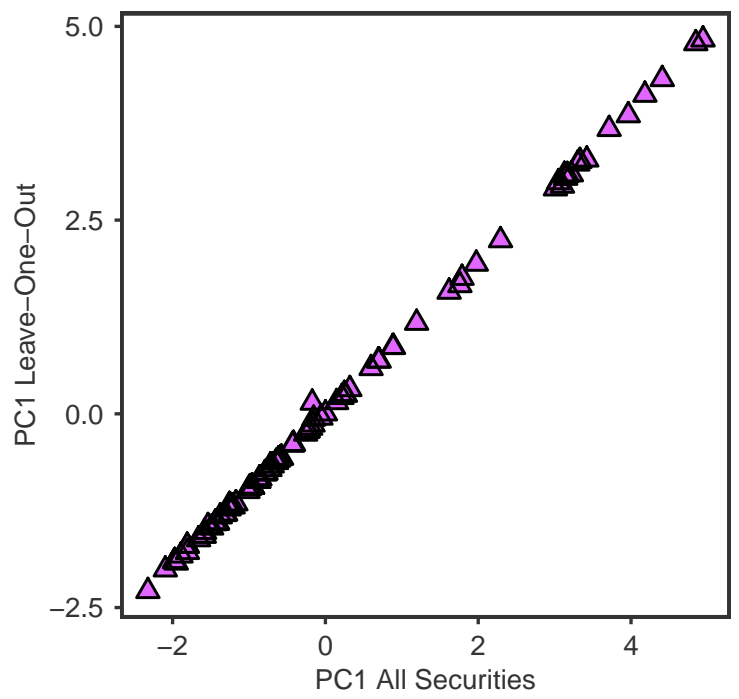

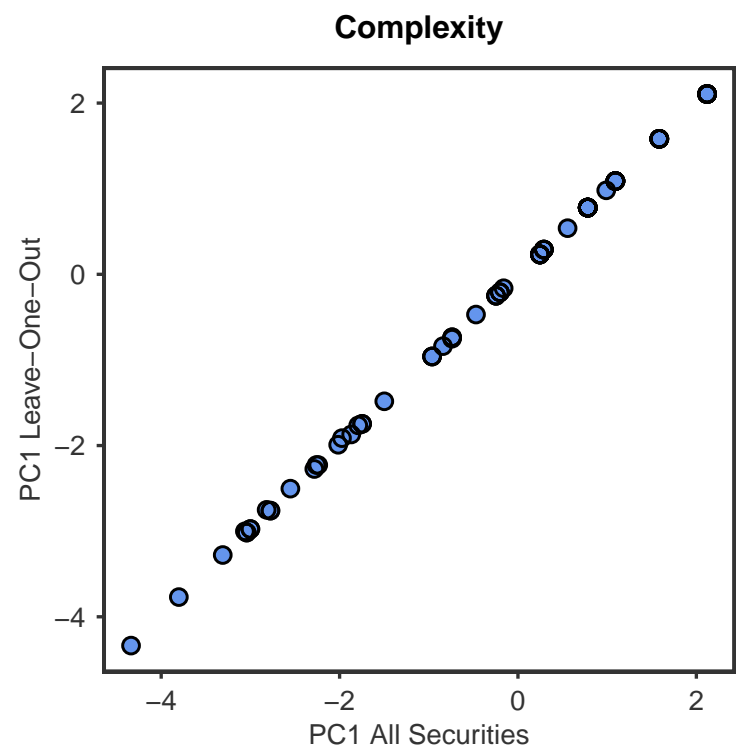

Figure A7: $f_{1}\left(x_{j}\right)$ plotted against $\hat{f}_{1}\left(x_{j}\right)$ for risk factors (top left), for complexity factors (top right), for risk and complexity factors (bottom). $75.8000,25.5709$, and 0.2784 in each setup respectively. 


\section{References}

Härdle, W. K. and L. Simar (2015). Applied multivariate statistical analysis (4 ed.). Berlin: Springer.

Kolenikov, S. and G. Angeles (2009). Socioeconomic status measurement with discrete proxy variables: Is principal component analysis a reliable answer? Review of Income and Wealth 55(1), 128-165. 


\section{SFB 649 Discussion Paper Series 2016}

For a complete list of Discussion Papers published by the SFB 649, please visit http://sfb649. wiwi.hu-berlin.de.

001 "Downside risk and stock returns: An empirical analysis of the long-run and short-run dynamics from the G-7 Countries" by Cathy Yi-Hsuan Chen, Thomas C. Chiang and Wolfgang Karl Härdle, January 2016.

002 "Uncertainty and Employment Dynamics in the Euro Area and the US" by Aleksei Netsunajev and Katharina Glass, January 2016.

003 "College Admissions with Entrance Exams: Centralized versus Decentralized" by Isa E. Hafalir, Rustamdjan Hakimov, Dorothea Kübler and Morimitsu Kurino, January 2016.

004 "Leveraged ETF options implied volatility paradox: a statistical study" by Wolfgang Karl Härdle, Sergey Nasekin and Zhiwu Hong, February 2016.

005 "The German Labor Market Miracle, 2003 -2015: An Assessment" by Michael C. Burda, February 2016.

006 "What Derives the Bond Portfolio Value-at-Risk: Information Roles of Macroeconomic and Financial Stress Factors" by Anthony H. Tu and Cathy Yi-Hsuan Chen, February 2016.

007 "Budget-neutral fiscal rules targeting inflation differentials" by Maren Brede, February 2016.

008 "Measuring the benefit from reducing income inequality in terms of GDP" by Simon Voigts, February 2016.

009 "Solving DSGE Portfolio Choice Models with Asymmetric Countries" by Grzegorz R. Dlugoszek, February 2016.

010 "No Role for the Hartz Reforms? Demand and Supply Factors in the German Labor Market, 1993-2014" by Michael C. Burda and Stefanie Seele, February 2016.

011 "Cognitive Load Increases Risk Aversion" by Holger Gerhardt, Guido P. Biele, Hauke R. Heekeren, and Harald Uhlig, March 2016.

012 "Neighborhood Effects in Wind Farm Performance: An Econometric Approach" by Matthias Ritter, Simone Pieralli and Martin Odening, March 2016.

013 "The importance of time-varying parameters in new Keynesian models with zero lower bound" by Julien Albertini and Hong Lan, March 2016.

014 "Aggregate Employment, Job Polarization and Inequalities: A Transatlantic Perspective" by Julien Albertini and Jean Olivier Hairault, March 2016.

015 "The Anchoring of Inflation Expectations in the Short and in the Long Run" by Dieter Nautz, Aleksei Netsunajev and Till Strohsal, March 2016.

016 "Irrational Exuberance and Herding in Financial Markets" by Christopher Boortz, March 2016.

017 "Calculating Joint Confidence Bands for Impulse Response Functions using Highest Density Regions" by Helmut Lütkepohl, Anna StaszewskaBystrova and Peter Winker, March 2016.

018 "Factorisable Sparse Tail Event Curves with Expectiles" by Wolfgang K. Härdle, Chen Huang and Shih-Kang Chao, March 2016.

019 "International dynamics of inflation expectations" by Aleksei Netšunajev and Lars Winkelmann, May 2016.

020 "Academic Ranking Scales in Economics: Prediction and Imdputation" by Alona Zharova, Andrija Mihoci and Wolfgang Karl Härdle, May 2016.

\section{SFB 649, Spandauer Straße 1, D-10178 Berlin http:/ / sfb649.wiwi.hu-berlin.de}

This research was supported by the Deutsche Forschungsgemeinschaft through the SFB 649 "Economic Risk".

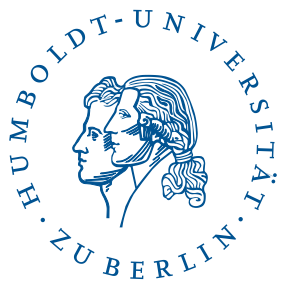




\section{SFB 649 Discussion Paper Series 2016}

For a complete list of Discussion Papers published by the SFB 649, please visit http://sfb649.wiwi.hu-berlin.de.

021 "CRIX or evaluating blockchain based currencies" by Simon Trimborn and Wolfgang Karl Härdle, May 2016.

022 "Towards a national indicator for urban green space provision and environmental inequalities in Germany: Method and findings" by Henry Wüstemann, Dennis Kalisch, June 2016.

023 "A Mortality Model for Multi-populations: A Semi-Parametric Approach" by Lei Fang, Wolfgang K. Härdle and Juhyun Park, June 2016.

024 "Simultaneous Inference for the Partially Linear Model with a Multivariate Unknown Function when the Covariates are Measured with Errors" by Kun Ho Kim, Shih-Kang Chao and Wolfgang K. Härdle, August 2016.

025 "Forecasting Limit Order Book Liquidity Supply-Demand Curves with Functional AutoRegressive Dynamics" by Ying Chen, Wee Song Chua and Wolfgang K. Härdle, August 2016.

026 "VAT multipliers and pass-through dynamics" by Simon Voigts, August 2016.

027 "Can a Bonus Overcome Moral Hazard? An Experiment on Voluntary Payments, Competition, and Reputation in Markets for Expert Services" by Vera Angelova and Tobias Regner, August 2016.

028 "Relative Performance of Liability Rules: Experimental Evidence" by Vera Angelova, Giuseppe Attanasi, Yolande Hiriart, August 2016.

029 "What renders financial advisors less treacherous? On commissions and reciprocity" by Vera Angelova, August 2016.

030 "Do voluntary payments to advisors improve the quality of financial advice? An experimental sender-receiver game" by Vera Angelova and Tobias Regner, August 2016.

031 "A first econometric analysis of the CRIX family" by Shi Chen, Cathy YiHsuan Chen, Wolfgang Karl Härdle, TM Lee and Bobby Ong, August 2016.

032 "Specification Testing in Nonparametric Instrumental Quantile Regression" by Christoph Breunig, August 2016.

033 "Functional Principal Component Analysis for Derivatives of Multivariate Curves" by Maria Grith, Wolfgang K. Härdle, Alois Kneip and Heiko Wagner, August 2016.

034 "Blooming Landscapes in the West? - German reunification and the price of land." by Raphael Schoettler and Nikolaus Wolf, September 2016.

035 "Time-Adaptive Probabilistic Forecasts of Electricity Spot Prices with Application to Risk Management." by Brenda López Cabrera , Franziska Schulz, September 2016.

036 "Protecting Unsophisticated Applicants in School Choice through Information Disclosure" by Christian Basteck and Marco Mantovani, September 2016.

037 "Cognitive Ability and Games of School Choice" by Christian Basteck and Marco Mantovani, Oktober 2016.

038 "The Cross-Section of Crypto-Currencies as Financial Assets: An Overview" by Hermann Elendner, Simon Trimborn, Bobby Ong and Teik Ming Lee, Oktober 2016.

039 "Disinflation and the Phillips Curve: Israel 1986-2015" by Rafi Melnick and Till Strohsal, Oktober 2016.

\section{SFB 649, Spandauer Straße 1, D-10178 Berlin http:/ / sfb649.wiwi.hu-berlin.de}

This research was supported by the Deutsche Forschungsgemeinschaft through the SFB 649 "Economic Risk".

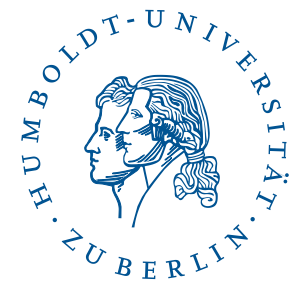




\section{SFB 649 Discussion Paper Series 2016}

For a complete list of Discussion Papers published by the SFB 649, please visit http://sfb649.wiwi.hu-berlin.de.

040 "Principal Component Analysis in an Asymmetric Norm" by Ngoc M. Tran, Petra Burdejová, Maria Osipenko and Wolfgang K. Härdle, October 2016.

041 "Forward Guidance under Disagreement - Evidence from the Fed's Dot Projections" by Gunda-Alexandra Detmers, October 2016.

042 "The Impact of a Negative Labor Demand Shock on Fertility - Evidence from the Fall of the Berlin Wall" by Hannah Liepmann, October 2016.

043 "Implications of Shadow Bank Regulation for Monetary Policy at the Zero Lower Bound" by Falk Mazelis, October 2016.

044 "Dynamic Contracting with Long-Term Consequences: Optimal CEO Compensation and Turnover" by Suvi Vasama, October 2016.

045 "Information Acquisition and Liquidity Dry-Ups" by Philipp Koenig and David Pothier, October 2016.

046 "Credit Rating Score Analysis" by Wolfgang Karl Härdle, Phoon Kok Fai and David Lee Kuo Chuen, November 2016. 